\title{
Dispersion of axially symmetric waves in fluid-filled cylindrical shells
}

\author{
Bao, X.L.; Überall, H.; Raju, P. K.; Ahyi, A. C.; Bjørnø, Irina; Jensen, Leif Bjørnø
}

Published in:

Acoustical Society of America. Journal

Link to article, DOI:

$10.1121 / 1.429206$

Publication date:

2000

Document Version

Publisher's PDF, also known as Version of record

Link back to DTU Orbit

Citation (APA):

Bao, X. L., Überall, H., Raju, P. K., Ahyi, A. C., Bjørnø, I., \& Jensen, L. B. (2000). Dispersion of axially symmetric waves in fluid-filled cylindrical shells. Acoustical Society of America. Journal, 107(5), 2847-2848.

https://doi.org/10.1121/1.429206

\section{General rights}

Copyright and moral rights for the publications made accessible in the public portal are retained by the authors and/or other copyright owners and it is a condition of accessing publications that users recognise and abide by the legal requirements associated with these rights.

- Users may download and print one copy of any publication from the public portal for the purpose of private study or research.

- You may not further distribute the material or use it for any profit-making activity or commercial gain

- You may freely distribute the URL identifying the publication in the public portal

If you believe that this document breaches copyright please contact us providing details, and we will remove access to the work immediately and investigate your claim 


\title{
Session $3 \mathrm{aAA}$
}

\section{Architectural Acoustics: Acoustical Design of Learning Spaces}

\author{
Brandon D. Tinianow, Chair \\ Acoustical Laboratory, Johns Manville, 10100 West Ute Avenue, Littleton, Colorado 80162
}

Invited Papers

9:00

3aAA1. Acoustical design of learning spaces. Paul Tan (Pelton Marsh Kinsella, 1420 W. Mockingbird Ln., \#400, Dallas, TX 75247, tanpl@c-b.com)

Learning spaces in the past 100 years have evolved from chalkboards and printed media to real-time digital distant learning centers equipped with the latest in communications and multimedia technologies. As a result, the modern learning space bears little semblance with its archetypal shoe-box classroom most are accustomed to. What are the criteria for designing a fully integrated learning environment, wherein human factors and technological systems function symbiotically within a carefully optimized space? This paper will seek to explore the impact of room acoustics, sound isolation, building systems, and equipment noise on the functionality and success of the modern learning environment through selected design cases.

\section{9:20}

3aAA2. Classroom acoustics: The effects of background noise and room-finish materials on speech intelligibility. Gary Siebein (Dept. of Architecture, Univ. of Florida, P.O. Box 115702, Gainesville, FL 32611-5702)

This paper will review recent research conducted at the University of Florida defining conditions in actual school classrooms that contribute to speech intelligibility. Many classroom settings were observed to determine how communication paths among teachers and students occurred in modern classrooms. A survey of classrooms was conducted with measurements of background noise levels, STI, and reverberation time made in the rooms at locations corresponding to those found in actual rooms. A computer model and a physical model of a typical classroom were constructed to further study classroom acoustic situations. A second-order curve was found relating RASTI to background noise levels (as a result of air-conditioning system noise) and distance from the teacher. The effects of room-finish materials played a secondary role in increasing RASTI once background noise levels of NC 32 or less were achieved.

\section{9:40}

3aAA3. Designing and building for quiet in a school for deaf children. John Guenther, Marcus Adrian (Mackey Mitchell and Assoc., St. Louis, MO), J. T. Weissenburger (Eng. Dynam. Intl., Inc., St. Louis, MO), and William Clark (Central Inst. for the Deaf, 818 S. Euclid, St. Louis, MO 63110)

Central Institute for the Deaf (CID) recently completed construction of a new 42000 -sq. ft. school for deaf children. High priority was placed upon designing and building a facility that would provide ideal acoustic environments that fostered learning and auditory/ oral communication for students wearing powerful hearing aids or cochlear implants and teachers. A team composed of scientists, architects, and acoustical engineers was assigned the task of designing and building a school that would provide classroom environmental levels at or below the NC 20 contour, interclassroom attenuation exceeding $50 \mathrm{~dB}$, reverberation times on the order of $0.4 \mathrm{~s}$, and sound reinforcement for teachers' voices when facing the blackboard. In group spaces and in the hallways, higher noise levels and longer reverberation times were sought to provide students with experiences more like those faced in the real world. Challenges included a site bounded by a busy interstate highway and a medical center heliport. The team developed and implemented numerous unique acoustic treatments for the facility which are reviewed in the presentation. Although designed as a school for the deaf, the approaches are useful for designing any educational classroom environment. The school opened on 10 January 2000 and met all acoustic criteria.

\section{0:00}

3aAA4. Acoustical modeling and auralization as a design tool for a university concert hall renovation. Bennett M. Brooks (Brooks Acoust. Corp., 27 Hartford Turnpike, Vernon, CT 06066, bbrooks@brooks-acoustics.com)

A 400-seat university concert hall was notorious for its bad acoustics. Music faculty identified the worst problem with this educational facility as poor stage communication, or the difficulty that performers had hearing one another. The hall was modeled using the CATT-Acoustic software system. Good agreement was found between objective measurements and model predictions for the original hall. Also, the subjective quality of the auralized model was judged to be an accurate representation of the original hall 
by the faculty. These results provided confidence in the model as a design tool for hall renovations. Design decisions were made based on objective parameters, such as early reflection density, and also on the subjective quality of the sound, for a series of model configurations. The final design was largely realized and the renovated hall has been well received by students, faculty, and community. Sound samples illustrating auralized design options will be presented.

\title{
Contributed Papers
}

10:20

3aAA5. Seeking improved speech intelligibility in a university classroom. Dean R. Heerwagen and Paul D. Sampson (Univ. of Washington, Box 355720, Seattle, WA 98195-5720)

Speech intelligibility has been studied while systematically modifying an existing classroom. The "original" classroom had acceptable background noise levels, but excessive reverberation. Room modifications included installing a lowered ceiling and successive additions of absorption to the classroom walls. The unoccupied rhyme tests (RTs) at $1 \mathrm{kHz}$ progressed from an "original" $1.10 \mathrm{~s}$ to a "final" $0.53 \mathrm{~s}$. Modified rhyme tests (MRTs) were also conducted with a volunteer group of faculty and staff. For each room condition, MRTs were administered across a range of signal-to-noise ratio (SNR) conditions. The principal parameters of this study were the physical conditions of the room (as indicated by RT and U50 measures), the test scores, the SNRs for the tests, distances between a loudspeaker and each volunteer, whether the volunteer was a native English speaker, and whether the volunteer was normal hearing or hearing impaired. Statistical analyses of the data indicate that the most important determinant of test performance was the SNRs. The correlation between test scores and RTs is also significant, but less so. Additionally, correlations between test scores and native and non-native speakers and between test scores and those with and without hearing impairment were also significant.

\section{0:35}

3aAA6. Further acoustical analysis of infant/toddler rooms in daycare centers. Matthew V. Golden (Grad. Prog. in Acoust., Penn State Univ., P.O. Box 30, State College, PA 16804, golden@sabine.acs.psu.edu) and Tom Frank (Penn State Univ., University Park, PA 16802)

At the last ASA meeting, preliminary data was shared on the background noise in daycare centers. At this meeting, further research in the acoustical analysis of infant/toddler rooms in seven daycare centers will be discussed in relation to speech communication. Information concerning the collection and analysis of ambient noise, signal-to-noise ratios (SNRs), reverberation times (RT), and early decay time (EDT) will be shared. Comparisons will be made between the measurements and data collection techniques of ambient noise from dosimeters, sound level meters, and other long-term data acquisition techniques. Comparisons of the overall and octave band RTs and EDTs from each of the rooms in the study will be shown. Finally, the calculation of SNRs will be shown for both steadystate and dynamic signals. The steady-state SNR uses several different speech spectra compared to the occupied 1/3-octave band noise levels. The dynamic SNRs are calculated using microphones placed at both the caregiver and the infant or toddler. [Work supported by PHS/NIH (1-R01HD31540-01A2) Otitis Media, Behavior and Attention in Daycare.]

\section{0:50}

3aAA7. A classroom acoustic model to evaluate prescriptive options to meet a performance standard. Richard D. Godfrey (Integrex, Bldg. 75, 2790 Granville Rd., Granville, OH 43023, dick.godfrey@owenscorning.com)

A standard to prescribe the acoustical performance of classroom spaces is now under development. As drafted, the standard will contain performance requirements, and many members of the working group would like to include prescriptive requirements as well. In order to make these two approaches consistent, an acoustical model of the classroom space is needed to predict the effects of component performance on the overall acoustic performance of the space. A model based on classical acoustics has been developed which allows the designer to select components performance characteristics from menus of measured performance. These input data are entered into an energy balance which predicts the classroom sound pressure level as a function of position in the room and the reverberation time. These performance characteristics are then compared to various metrics being considered by the working group. In this paper the formulation of the model will be described, and proposed prescriptive options will be evaluated for consistence with the performance metrics.

\section{Session $3 \mathrm{aAB}$}

\section{Animal Bioacoustics, Psychological and Physiological Acoustics and Speech Communication: Cognitive Aspects of Complex Sound Perception in Animals}

\author{
Robert J. Dooling, Chair \\ Psychology Department, University of Maryland, College Park, Maryland 20742
}

Chair's Introduction-8:30

\section{Invited Papers}

8:35

\begin{abstract}
3aAB1. Complex sound source determination: Behavioral studies on goldfish. R. R. Fay (Parmly Hearing Inst. and Dept. of
\end{abstract} Psych., Loyola Univ. of Chicago, Chicago, IL 60626)

A series of experiments is reported on complex sound perception in goldfish using classical respiratory conditioning and a stimulus generalization paradigm. In general, animals are initially conditioned to a simple or complex sound, and then tested for generalization to novel sounds that differ systematically from the conditioning sound along one or more stimulus dimensions. Generalization 
gradients to the novel sound set indicate what sounds are perceived as similar or dissimilar, and what features of sounds are analyzed or attended to during conditioning and generalization testing. So far, these kinds of experiments have demonstrated perceptual dimensions in goldfish that resemble the dimensions of pure tone pitch, complex pitch, timbre, and roughness as perceived by humans. In addition, several experiments have revealed auditory stream segregation in goldfish that resembles that demonstrated for human listeners. It is concluded that goldfish share with humans a sense of hearing that is primitive for vertebrates, and is likely shared with most other vertebrate species. At a qualitative level, at least, goldfish know what we know about sounds and their sources. [Work supported by the NIH, NIDCD.]

\section{9:00}

3aAB2. Target tracking by echolocation in a dynamic auditory scene. Cynthia F. Moss (Dept. of Psych., Prog. in Neurosci. and Cognit. Sci., Univ. of Maryland, College Park, MD 20742) and Annemarie Surlykke (Odense Univ., DK-5230 Denmark)

The echolocating bat transmits ultrasonic vocalizations as it flies, and it perceives a dynamic, 3-D representation of the world from echoes of its sonar signals. We hypothesize that the bat's auditory system organizes dynamic echo input to segregate and track multiple reflecting sources in the environment, and these perceptual processes depend on the features of the animal's sonar signals. Our research here focuses on the bat's timing of sonar cries to enhance spatial tracking of objects in a dynamic auditory scene. Using two distinct behavioral methods, we quantified features of the bat's vocal signals in target tracking tasks. In a psychophysical study, bats were trained to track changing echo delays of phantom targets. The bat's sonar cries were digitized, electronically delayed, and played back to the animal, simulating targets that approached or disappeared at different rates. Features of the animal's sonar vocalizations were measured for each trial and summarized across conditions. In another study, high-speed synchronized video and audio recordings provided measures of the bat's sonar behavior as it tracked and intercepted tethered insects that were either stationary, moving smoothly, moving erratically, displaced abruptly, or positioned near obstacles. The data illustrate acoustic control for tracking in a dynamic auditory scene.

3aAB3. The evasive responses of praying mantids and tiger beetles to complex ultrasonic pulse trains produced by echolocating bats. David D. Yager (Dept. of Psych., Univ. of Maryland, College Park, MD 20742, dy5@umail.umd.edu)

Two highly visual diurnal insect predators, praying mantids and tiger beetles, become the prey of echolocating bats at night. Although very distant phylogenetically, the two insects have evolved similar ultrasound-triggered behavioral responses to help them evade capture. The tiger beetle ears have a conventional tympanate design with sharp tuning at $30-35 \mathrm{kHz}$ and directional capabilities. The mantis peripheral auditory system is highly unconventional with a single cyclopean ear in the metathorax that apparently sacrifices directionality for increased sensitivity. There are six to eight pairs of ascending auditory interneurons in the mantis thorax. Even though it slows response time, auditory processing in the brain is required for the evasive behavior, i.e., it is not a simple reflex. The tiger beetles behavioral response to ultrasound is similar, but there appears to be no system of large ascending interneurons. In both insects, the nocturnal ultrasound-triggered evasive behaviors probably evolved from diurnal defensive displays triggered by visual stimuli. Thus, the evolutionary innovation may not have been extensive new circuitry, but, rather, a context-dependent switch that links the behavior to vision during the day and to hearing at night.

\section{9:50}

3aAB4. Discrimination of temporal features in complex sounds by the bullfrog, Rana catesbeiana. Andrea M. Simmons (Dept. of Psych., Brown Univ., Providence, RI 02912)

The advertisement calls of the sympatric species the bullfrog (Rana catesbeiana) and the green frog (Rana clamitans) differ in duration and first harmonic periodicity. Sensitivity of male bullfrogs to complex sounds differing in these acoustic attributes was examined in the field using the evoked-calling technique. In experiment 1 (duration series), males were presented with a series of synthetic signals, all with the spectral structure of the bullfrog's advertisement call, but varying in duration along a continuum from bullfrog-like to green-frog-like. In experiment 2 (pitch series), signals were of bullfrog-like duration, but varied in first harmonic periodicity in steps from bullfrog-like to green-frog-like. The number and latencies of the males' evoked vocal responses differed significantly to the exemplars within both series. The forms of the discrimination functions suggest that males perceived these acoustic features as distinct categories. Discrimination was invariant with sound-pressure level over a 30-dB range. These data show that bullfrogs use temporal features to distinguish between conspecific and heterospecific vocalizations, and may achieve this by a process similar to categorical perception as defined in other animals.

\section{0:15-10:30 Break}

\section{0:30}

3aAB5. Why macaque screams differ. Harold Gouzoules and Sarah Gouzoules (Dept. of Psych. and Yerkes Primate Ctr., Emory Univ., Atlanta, GA 30322, psyhg@emory.edu)

Screams of four species of macaques (Macaca mulatta, M. nemestrina, M. nigra, M. arctoides) were compared for similarities and differences with respect to predictions of Morton's motivation-structural rules [Morton, Am. Nat. 111, 855-869 (1977)]. Screams from victims of attack that involved contact aggression (pulling, pushing, slapping, grappling, and biting) from a higher-ranking opponent were examined. For each species, 100 screams from females three years of age or older were digitized and acoustic features 
of each call measured. Discriminant function analysis was used to determine whether or not the 400 vocalizations could be assigned to the correct caller species on the basis of their acoustic structure. Calls were assigned to the correct species at a significantly higher rate $(93.5 \%)$ than expected by chance. Each of the four macaque species used acoustically distinct screams in a shared context. While the differences in the species' vocalizations suggest no simple correlation between immediate context and the acoustic forms of screams, there was general correspondence between the structure predicted by motivation-structural rules and inferences about the internal state of the vocalizer derived from the typical intensity of aggressive patterns that characterize each of the four species.

\section{$10: 55$}

3aAB6. Contributions of nonhuman animal models to understanding human speech perception. Keith R. Kluender (Dept. of Psych., Univ. of Wisconsin, 1202 West Johnson St., Madison, WI 53706)

Broadly speaking, nonhuman animal models contribute to understanding speech perception by humans in two ways-by analogy and by homology. The former is generally easier and examples are more abundant. Because demonstrating homology requires deeper explication of underlying mechanisms, claims can be more precarious but carry potentially greater explanatory payoff. When studying nonhuman organisms as an analogy, the emphasis is typically upon how animal physiological or behavioral processes have adapted to fulfill requirements of particular ecological niches. By contrast, study of animals as homology often violates ecology in search of common underlying processes, and the animal becomes a method more than an object of study. Examples of findings for animal analogies and homologies will be reviewed. Data will be presented from experiments in which nonhuman subjects play the role of homology in revealing both foundational sensory processes and more plastic processes of perceptual development. Animal models provide advantages for describing sensory representation of speech unadulterated by effects of experience, and animal models allow control over experience permitting better characterization of processes through which experience shapes perception. Nonhuman animal models are providing important insights into general processes of audition and learning essential to human speech perception. [Work supported by NIH and NSF.]

\section{Contributed Papers}

\section{$11: 20$}

3aAB7. 3D models of lateral line excitation patterns during prey acquisition by Lake Michigan mottled sculpin. Sheryl Coombs (Parmly Hearing Inst., Loyola Univ. of Chicago, IL 60626) and James Finneran (SPAWAR Systems Ctr., San Diego, CA 92152-6505)

One of the biggest impediments to understanding how fish acquire information through their lateral line sensory system is the inherent difficulty in adequately specifying and measuring hydrodynamic stimuli, which can vary in frequency, amplitude, and phase along the sensory surface of the fish. Even theoretically simple stimuli, like dipole sources, can create rather spatially complex water motions. The peripheral excitation pattern after the stimulus field has been transduced can be even more complex and depends on a number of factors, including the density of the fish and the amplitude and direction of its body motion in the field, the number, type, and spatial distribution of lateral line end organs on the fish, the axis of source vibration, and the distance and orientation of the fish with respect to the source. We have developed a 3D computational model that takes these factors into account and is used to (1) compare the pretransduction distribution of the stimulus (flow) field along the sensory surface of the fish to the post-transduction excitation pattern of primary afferent fibers and (2) describe how excitation patterns change during actual approach pathways taken by sculpin when responding to preylike (dipole) sources.

\section{1:35}

3aAB8. Cortical responses in rats to periodic frequency-modulated sounds. Itzel Orduna, Eduardo Mercado III, Daphna Shohamy, Mark A. Gluck (CMBN, Rutgers Univ., 197 University Ave., Newark, NJ 07102), and Michael M. Merzenich (Coleman Lab., UCSF, San Francisco, CA 94143)

Spectral variations over time are a common characteristic of naturally occurring sounds. They constitute a prevalent feature in communication signals in several species. Studies of mammals have shown that auditory cortex neurons respond to single frequency-modulated (FM) sweeps and that most responses are selective for sweep direction and/or rate. Researchers have also used trains of FM sweeps to estimate spectrotemporal receptive fields in auditory cortex. In the present study, microelectrode recordings were used to explore how the auditory cortex responds to trains of FM sweeps in anesthetized rats. Maps of 20-60 penetrations were made for each subject. Sweep frequencies ranged from 1-16 kHz with FM rates ranging from 4-24 octaves/s and repetition rates from 2-24 sweeps/s. Both down-sweeps and up-sweeps were presented. Neuronal responses were analyzed in terms of onset, offset, oscillatory and directionally selective properties. Several types of responses predominated. Most units responded to sound onset. In contrast, responses to signal offsets were rare. Oscillatory responses were typically evoked only for repetition rates less than 12 sweep/s. Directionally selective responses were limited to these rates. These results indicate that oscillatory responses in the auditory cortex are limited to low repetition rate stimuli, and that directional selectivity depends on repetition rate. [Work supported by NSF.]

\section{1:50}

3aAB9. Plasticity of spectrotemporal sensitivities in auditory cortex. Eduardo Mercado III, Daphna Shohamy, Itzel Orduna, Mark A. Gluck (CMBN, Rutgers Univ., 197 University Ave., Newark, NJ 07102), and Michael M. Merzenich (Coleman Lab., UCSF, San Francisco, CA 94143)

Response characteristics of auditory cortex can be altered by repeatedly pairing sounds with basal forebrain stimulation [M. P. Kilgard and M. M. Merzenich, Science 279, 1714-1718 (1998)]. Although many neurons in auditory cortex respond most strongly to time-varying sounds, most studies of stimulation-induced plasticity have focused on changes in responses to tone pips. We examined stimulation-induced changes in neuronal sensitivities to frequency-modulated sweep trains (bandwidth $=2-16$ $\mathrm{kHz}$, duration $=1 \mathrm{~s}$, sweep rates $=4-24$ octaves $/ \mathrm{s}$, repetition rates $=2-24$ sweeps/s). Adult rats received electrical stimulation of basal forebrain paired with 1-10 varieties of sweep trains, 300-500 times per day, for 9-16 days. Some sounds were presented in combination with bandlimited 
Gaussian noise. After stimulation, neuronal responses were recorded from 20-80 sites in the auditory cortex of each rat. The spectrotemporal sensitivities of auditory cortical neurons were dramatically altered in stimulated rats. Changes in response characteristics were not straightforwardly related to features of the sounds that had been paired with stimulation.
Certain sounds that normally evoked responses correlated with sweep repetition rate in control rats tended to evoke either aperiodic or uncorrelated periodic responses from neurons of stimulated rats. Current theories of auditory cortical plasticity do not account for these results. [Work supported by MBRS, NIMH, NSF.]

THURSDAY MORNING, 1 JUNE 2000

ENGLISH ROOM, 9:00 TO 11:00 A.M.

\title{
Session 3aAO
}

\section{Acoustical Oceanography: Comparisons of Acoustical and Conventional Measurements of Ocean Thermohaline Structures}

\author{
Brian D. Dushaw, Chair \\ Applied Physics Laboratories, University of Washington, 1013 NE 42nd Street, Seattle, Washington 98105-6698
}

Chair's Introduction-9:00

Invited Paper

9:05

\begin{abstract}
3aA01. Tomographic maps of the New England Shelfbreak Front. Ching-Sang Chiu (Code OC/Ci, Dept. of Oceanogr., Naval Postgrad. School, Monterey, CA 93943 chiu @nps.navy.mil), James F. Lynch, and Glen Gawarkiewicz (Woods Hole Oceanogr. Inst., Woods Hole, MA 02543)

In July 1996, an integrated acoustic-oceanographic experiment was carried out in the Mid Atlantic Bight south of New England to study the oceanographic structure of the shelfbreak front and its effects on sound propagation. The experiment employed a suite of acoustic and oceanographic sensors including sound sources on the slope, vertical hydrophone arrays on the shelf, and a SeaSoar (towed CTD) that provided several volumetric surveys of the frontal zone. In order to obtain accurate tomographic maps for this complex shelf-slope environment, an inverse scheme that can properly handle strong mode coupling was developed. In the formulation, a variation in the modal travel time is expressed as a function of the evolution history and coupling history of the modes associated with the "background" ocean and changes in these histories due to sound speed changes. The inversion of the observed modal travel times is accomplished using nonlinear least-squares estimations. In this presentation, the quality of these tomographic maps is discussed using results from a resolution-variance analysis. Additionally, the data and resolution kernels of the tomographic measurements are compared to those of the SeaSoar measurements to illustrate that the two independent data sets are highly complementary to each other.
\end{abstract}

\section{Contributed Papers}

9:35

3aAO2. Acoustic monitoring of oceanographic features and processes in the Arctic Ocean. Konstantin Sabinin (N. N. Andreev Acoust. Inst., Shvernik St., Moscow, 117036, Russian Federation) and James Lynch (Woods Hole Oceanogr. Inst., Woods Hole, MA 02543)

Acoustic transmissions can be used to monitor two of the most important features of the Arctic Ocean, namely, the heat content of the Atlantic water and the upper ocean salinity. In this presentation, we will first discuss the optimal configuration of an array that might be used for heat content studies. We will then look at the newer concept of monitoring the upper ocean salinity in the Arctic Ocean using acoustics, which we have dubbed "acoustic halinometry." Both the basic concepts of halinometry and the configuration of a possible experimental array will be discussed. In particular, we will show the results of numerically simulating an acoustic halinometry experiment using data from large-scale Arctic oceanographic surveys conducted during 1973-1979. We will also look at the integration of the acoustics with conventional measurements in a "combined monitoring" array concept. This combined monitoring concept will be applied via simulation to one of the primary connections to the Arctic Ocean, the Bering Strait.
9:50

3aAO3. A comparison of acoustic thermometry, XBT, TOPEX, and HOT observations of ocean temperature in the northeast Pacific. Brian D. Dushaw and the ATOC Group ${ }^{\text {a) }}$ (Appl. Phys. Lab., Univ. of Washington, 1013 NE 40th St., Seattle, WA 98105-6698)

Time series of temperature have been measured acoustically in the northeast Pacific as part of the Acoustic Thermometry of Ocean Climate (ATOC) project. These time series are compared with other available data types. The acoustic time series of transmissions from the California and Kauai acoustic sources were obtained during 1996-1999. As a result of marine mammal protocols, the time series are intermittent; the California source was turned off in Fall 1998. Assuming that variations in sea-surface height observed by TOPEX/POSEIDON are caused by thermal expansion, the amplitude of the annual cycle of heat content derived from altimetry is larger than that found by the acoustic data, Levitus climatology, and monthly maps of ocean temperature from XBTs of opportunity. The heat content "anomalies" determined by the XBT maps are comparable in size to the differences between the XBT and acoustically derived heat content. A variety of problems with the XBT sampling may account for these 
differences. The 12-year time series of temperature derived from the Hawaiian Ocean Time series (HOT) data highlights the mesoscale noise in single-point sampling. However, thermal variability at 100-day time scales is observed in the acoustic data obtained between Hawaii and California using the Kauai source. Acoustic thermometry is complementary to altimetry and hydrography. ${ }^{a)}$ The Acoustic Thermometry of Ocean Climate
(ATOC) Group is: A. B. Baggeroer and C. Wunsch (MIT); D. Menememlis (JPL); T. G. Birdsall, K. Metzger (Univ. of Mich.); C. Clark (Cornell Univ.); J. A. Colosi (WHOI); B. D. Cornuelle, M. Dzieciuch, W. Munk, P. F. Worcester (SIO); D. Costa (Univ. of Calif., Santa Cruz); B. D. Dushaw, B. M. Howe, J. A. Mercer, R. C. Spindel (APL-Univ. of Wash.); A. M. G. Forbes (CSIRO, Hobart).

10:05-11:00

Panel Discussion

THURSDAY MORNING, 1 JUNE 2000

FRENCH ROOM, 8:30 TO 10:45 A.M.

Session 3aBB

Biomedical Ultrasound/Bioresponse to Vibration: Lithotripsy

\author{
Robin O. Cleveland, Chair \\ Aerospace and Mechanical Engineering, Boston University, 110 Cummington Street, Boston, Massachusetts 02215
}

\title{
Contributed Papers
}

8:30

3aBB1. Study of weak underwater shock waves generated by Ho:YAG pulse laser beam. S. Hamid, R. Hosseini (Shock Wave Res. Ctr., Inst. of Fluid Sci., Tohoku Univ., 2-1-1 Katahira, Aoba, Sendai 980-8577, Japan, hosseini@ceres.ifs.tohoku.ac.jp), Takayuki Hirano, Osamu Onodera, and Kazuyoshi Takayama (Tohoku Univ., Sendai, Japan)

For medical application of underwater shock waves as a less-invasive approach, a reliable micro shock wave source is required. The present paper reports progress in production of underwater micro shock waves by direct irradiation of pulse laser beam. Energy source was a Q-switched Ho:YAG laser (Nippon Infrared Industries Co. Ltd.) with $91 \mathrm{~mJ} / \mathrm{pulse}$ energy measured at the end of a $0.60-\mathrm{mm}$ diameter glass optical fiber, pulse duration of $200 \mathrm{~ns}$, and wavelength of $2.1 \mu \mathrm{m}$. The laser beam was focused and transmitted through the optical fiber. The generation and propagation of underwater shock waves from the roughened end of the fiber were quantitatively visualized by double-exposure holographic interferometry. Sequential flow visualization revealed that plasma generated by the laser beam drove spherical shock waves. The diameter of plasma region increased, then reduced and detached from the fiber end. Heatinduced flow in front of the fiber vanished after $100 \mathrm{~ms}$. Peak overpressures were measured at various stand-off distances. The weak shock waves produced by this method have potential to be applied for precise medical procedures such as revascularization in neurosurgery.

\section{8:45}

3aBB2. The impact of high-dose lithotripsy on renal structure and function. Lynn R. Willis (Dept. of Pharmacology and Toxicology, Indiana Univ. School of Medicine, 635 Barnhill Dr., Indianapolis, IN 46202, willis1@iupui.edu), Andrew P. Evan, Bret A. Connors, Philip Blomgren, and James E. Lingeman (Indiana Univ. School of Medicine, Indianapolis, IN 46202)

These studies characterized the effects of normal and high "doses" of shock waves on renal structure and function in healthy and compromised kidneys of young, anesthetized pigs. A "normal" dose of 2000 shock waves (24 kV, unmodified HM3 lithotripter) to one kidney produced lesions comprising about $8 \%$ of functional renal mass, transiently reduced renal blood flow and glomerular filtration rate (GFR) in both kidneys, and reduced regional perfusion in shocked kidneys. High doses of shock waves to one kidney ( 8000 shocks at $24 \mathrm{kV}$ ) produced larger lesions (about 14\%), intensified the initial vasoconstriction in both kidneys, and sustained the reduction of GFR in the shocked kidneys for at least $24 \mathrm{~h}$ after treatment. The administration of 2000 shock waves $(24 \mathrm{kV})$ to pyelonephritic (compromised) kidneys exaggerated the structural/functional impairment by inducing large lesions and intense vasoconstriction normally seen only after 8000 shock waves in healthy kidneys. We conclude that both shock wave number and preexisting renal disease increase the severity of tissue injury and functional impairment produced by shock wave lithotripsy. Since renal ischemia and inflammation occur in association with such injury, subsequent renal scarring and permanent loss of functional renal mass may also be related to shock wave dosage.

\section{9:00}

3aBB3. Effect of shock waves on cytoskeleton of human renal cell carcinoma. S. Moosavi Nejad, Makota Satoh, Naomasa Ioritani, and Seiichi Orikasa (Dept. of Urology, School of Medicine, Tohoku Univ., Seiryo-machi, Aoba, Sendai, 980-8574 Japan)

The first clinical extracorporeal shock wave lithotripsy (ESWL) in early 1980 revolutionized the surgical management of urolithiasis. Since then, studies have been under way to discover additional uses of ESWL in other areas of medicine including treatment of cancer. However, the shock wave tissue interactions have not yet completely been understood. To analyze in vitro subsequent alteration of cell cytoskeleton, cultured human renal carcinoma cells were exposed to the underwater shock waves, generated by an experimental setup with $20 \mathrm{MPa}$ overpressure at the focal region. The cultured cells provided a three-dimensional lattice work of structural proteins (actin, tubulin, and vimentin) analogous to those observed in the solid tumor. Scanning electron microscopic examination revealed morphological changes of the treated cells located in the focal region. Cells were detached from the substratum and changed from the normal fibroblast-like shape into a spherical shape. Disorganization and disassembly of the fibrillar cytoskeletal proteins were observed in the treated cells by immunofluorescence microscopy. The detached cells were collected and recultured again. After $6 \mathrm{~h}$ of spreading on a culture dish, reorganization of those fibers in the treated cells was compared with control cells and no significant difference was found. 
localize these clusters. Such research has targeted radial, not translational motion. We investigate whether bubble translation due to radiation force is

3aBB4. SWL stone fragmentation in vitro is improved by slowing the SW delivery rate. David A. Lifshitz, James C. Williams, Jr., Andrew P. Evan, Drew L. Rietjens, James A. McAteer (Dept. of Anatomy and Cell Biol., Indiana Univ. School of Medicine, 635 Barnhill Dr., Indianapolis, IN 46202, mcateer@anatomy.iupui.edu), Michael R. Bailey, Lawrence A. Crum (Univ. of Washington, Seattle, WA 98105), and Oleg A. Sapozhnikov (Moscow State Univ., Moscow 119899, Russia)

Fast shock wave (SW) rates in lithotripsy (SWL) generate enhanced cavitation that could promote stone fragmentation. We tested the idea that SWL at the high end of clinical SW rate $(2 \mathrm{~Hz})$ acts to improve stone comminution. Model stones (Ultracal-30 cement) were exposed to SWs ( $20 \mathrm{kV}, 400 \mathrm{SWs}$ ) at $0.2,0.5,1$, and $2 \mathrm{~Hz}$ in a research electrohydraulic lithotripter. Fragmentation was assessed by measuring number, size, and projected surface area of the fragments. Stones treated at $0.2 \mathrm{~Hz}$ exhibited significantly greater fragmentation $(p<0.01)$ than stones at 1 or $2 \mathrm{~Hz}$, while fragmentation between 0.2 and $0.5 \mathrm{~Hz}$ was similar. Mean $\pm \mathrm{SEM}$ for fragment area increase was $370 \pm 53 \%$ at $0.2 \mathrm{~Hz}$ ( $n=10$ stones), 280 \pm 34 at $0.5 \mathrm{~Hz}(8), 130 \pm 31$ at $1 \mathrm{~Hz}(5)$, and $101 \pm 16$ at $2 \mathrm{~Hz}(20)$. This pronounced enhancement of fragmentation at very slow SW rate was unexpected. High-speed camera images of cavitation at solid objects show an increased bubble cloud at faster SW rates. The bubble cloud may interfere with transmission of acoustic energy to the stone surface. These in vitro data suggest the possibility that patient treatment at fast SW delivery rates may decrease the efficiency of stone comminution. [Work supported by NIH P01-DK43881.]

\section{9:30-9:45 Break}

\section{9:45}

3aBB5. Dynamics of lithotripter shock-wave-induced bubble oscillation in constrained media. Pei Zhong, Yufeng Zhou, and Songlin Zhu (Dept. of Mech. Eng. and Mater. Sci., Duke Univ., Box 90300, Durham, NC 27708, pzhong@acpub.duke.edu)

Rupture of small blood vessels is often observed in vivo following shock wave lithotripsy (SWL), and cavitation has been implicated as a potential mechanism for the injury. To understand more precisely the underlying mechanical process of the injury, the dynamics of SWL-induced bubble oscillation in constrained media have been investigated. Silicone tubes (0.3-1.5-mm inner diameter), filled with circulating saline solution containing $0.1 \%$ Albunex contrast agent, were immersed in castor oil and placed in the acoustic field of a Dornier XL-1 lithotripter. Bubble dynamics induced inside the silicone tubes were characterized using high-speed shadowgraph and passive cavitation detection via a $20-\mathrm{MHz}$ focused hydrophone. The result shows that the symmetric bubble oscillation, typical for SWL-induced cavitation in water, is largely disrupted due to the constraint of the silicone tube on bubble expansion, leading to an asymmetric elongation of the bubble along the tube axis. The subsequent collapse of the bubbles inside the silicone tube (or blood vessels) is therefore significantly weakened, and thus the potential to cause tissue injury. Additional experiments using hollow cellulose fibers suggest that the damage to small blood vessels may be caused by the rapid, large intraluminal expansion of the bubble. [Work supported by NIH.]

\section{0:00}

3aBB6. Bubble translation due to radiation force in SWL. Dahlia L. Sokolov, Michael R. Bailey, Lawrence A. Crum (Appl. Phys. Lab., Univ. of Washington, 1013 NE 40th St., Seattle, WA 98105, dsokolov@apl.washington.edu), and Oleg A. Sapozhnikov (Moscow State Univ., Moscow 119899, Russia)

The clustering of cavitation bubbles may lead to enhanced stone comminution and influence the extent of tissue damage during shock wave lithotripsy (SWL) treatment. Recent research has focused on changing the SWL pulse, or timing between pulses, to intensify or mitigate collapse or sufficiently large to influence cluster formation. The translational dynamics of a single spherical bubble were modeled according to the formulation proposed by Watanabe and Kukita [Phys. Fluids 5(11) (1993)]. After radius-time data were obtained using the Gilmore equation, translational motion was calculated by numerical integration of the Watanabe equation. Calculations were performed for a range of bubble sizes $\left(R_{0}=2-20 \mu \mathrm{m}\right)$ and pressure rise times $\left(10^{-9}-10^{-7} \mathrm{~s}\right)$. The results show that, during bubble growth and collapse induced by a single pulse or two pulses with microsecond delays, bubble translations are $\sim 0.1 \mathrm{~mm}$. Although bubble translation from a single pulse may not have a noticeable effect on bubble distribution, the effect may be cumulative for the $1000+$ shots fired during clinical SWL treatment. [Work supported by NIH DK43881, NSF, CRDF, and FIRCA.]

\section{0:15}

3aBB7. Simultaneous detection of acoustic and light emissions from cavitation bubbles in SWL. Thomas J. Matula, Michael R. Bailey, Paul R. Hilmo, and Lawrence A. Crum (Appl. Phys. Lab, Univ. of Washington, 1013 NE 40th St., Seattle, WA 98105, matula@apl.washington.edu)

A typical pulse in electrohydraulic shock wave lithotripsy (SWL) consists of an intense positive pressure pulse, followed by a longer negativepressure tail. Computer models of the bubble dynamics associated with such a pulse suggest that the positive pressure pulse compresses the bubble $\left(R_{\{0\}}=3-10 \mu \mathrm{m}\right)$ to a submicron size. The negative-pressure tail then causes the bubble to undergo a dramatic expansion, followed by an inertially dominated (presumably spherical) collapse hundreds of microseconds later. Acoustic and light emissions are generated at both collapses. We have examined the simultaneous acoustic and optical emission from a cavitation field generated by SWL in order to determine whether the sonoluminescence is principally due to the initial compression of the bubble, or the final inertial collapse. Using two confocal 1-MHz, piezoceramic hydrophones and a PMT mounted on a light-tight water-filled container, we have observed acoustic and light emission corresponding to both the compression and inertial collapse of the bubble field. Our initial results suggest that the light emission occurs most frequently during the initial bubble compression. These results may have implications for understanding the sphericity of the bubble dynamics produced in SWL. [Work supported by NIH and DARPA.]

\section{0:30}

3aBB8. Dynamic photoelastic study of the transient stress fields in solids during shock wave lithotripsy. Xufeng $\mathrm{Xi}$ and Pei Zhong (Dept. of Mech. Eng. and Mater. Sci., Duke Univ., Box 90300, Durham, NC 27708)

Photoelastic and shadowgraph imaging techniques were used to visualize shock wave propagation, evolution, and the resultant transient stress fields in solids during shock wave lithotripsy. In parallel, theoretical analysis of the wavefront evolution inside the solids was performed using the ray-tracing method. Excellent agreement between the theoretical prediction and experimental results was observed. Moreover, the effects of sample size and shape on wave evolution and associated stress fields induced inside the solids were evaluated, both theoretically and experimentally. Finally, stone fragmentation tests were carried out using stone phantoms of different geometry and size, and the characteristics of the damage patterns were documented. By correlating the stone fragmentation patterns with the behavior of different wave components induced in the target stones, it was found that while the damage cracks near the posterior surface of the sample were initiated by the reflected longitudinal tensile wave, the transmitted shear wave plays a critical role in crack extension. [Work supported by NIH.] 


\title{
Session 3aEA
}

\section{Engineering Acoustics: Transducers and Arrays}

\author{
Sung-Hwan Ko, Chair \\ College of Engineering, Seoul National University, Seoul 151-742, Korea
}

\section{Contributed Papers}

\section{9:00}

3aEA1. Characterization of a cosine-shaped horn. Jorge Arenas and Malcolm Crocker (Dept. of Mech. Eng., Auburn Univ., 201 Ross Hall, Auburn, AL 36849-5341, arenajo@eng.auburn.edu)

Several approaches to the problem of the acoustic wave propagation in a duct with a varying cross section have been described in the literature. It has been shown that a simple one-dimensional analysis gives accurate predictions of the sound propagation. In this research, the impedance of a cosine-shaped horn is obtained using the Wentzel-Kramers-Brillouin (WKB) approximation to solve the Webster wave equation and the results are compared with those obtained from a numerical solution of the equivalent system of nonlinear equations. A comparison of the throat impedance ratio is presented for similar exponential, conical, parabolic, and catenoidal horns with the same overall dimensions. The mouth of the horn is assumed to be terminated in an infinite baffle. In addition, since the device may be used as an impedance transformer, the results for the impedance ratio for two pipes of different areas joined by a cosine-shaped connector are compared with several well-known couplings. Such a cosine-shaped connector will act as a simple discontinuity when its length is short compared with a wavelength and as a transformer for acoustic impedance for higher frequencies.

\section{9:15}

3aEA2. Directivity patterns of rectangular pistons on prolate spheroids. J. E. Boisvert and A. L. Van Buren (Naval Undersea Warfare Ctr. Div. Newport, Newport, RI 02841-1708, boisvert@lego.npt.nuwc.navy.mil)

A general expression for the directivity of a rectangular piston arbitrarily located upon a rigid prolate spheroidal baffle is formulated. The piston is assumed to vibrate with uniform normal velocity, and Neumann boundary conditions are imposed in the solution of the problem. The formal solution is expressed in terms of a modal series representation in spheroidal wave functions, valid at any distance from the spheroid. The prolate spheroidal wave functions are obtained using computer programs that have been recently modified and extended to provide accurate values of the wave functions at high frequencies. Substitution of the limiting form of the radial wave functions (as $r$ approaches infinity) into the solution yields the piston far-field single element pattern (SEP). The SEPs for several different piston sizes, orientations, and locations on the spheroidal baffle are presented at various frequencies. [Work supported by ONR Code 321 and Naval Undersea Warfare Center In-house Laboratory Independent Research (ILIR) program.]

\section{9:30}

3aEA3. Recent transmit-mode experimental results on thicker injection molded 1-3 piezocomposite. Kim Benjamin (NAVSEA Newport, Undersea Warfare Ctr., Div. Code 2162, Newport, RI 02841)

Three transducers which utilize a new 25.4-mm (1.0-in.)-thick injection molded 1-3 piezocomposite as the active material have been fabricated and acoustically calibrated in the transmit mode. The study, which included a single, double, and quadruple layer assembly of 25.4-mm (1.0in.)-thick composite, indicates that considerable transmit source levels are possible over a very broad frequency range using this newly developed thicker material. Furthermore, the element design can easily be packaged into various two-dimensional array configurations. For the multilayer cases, the nulls present in the untuned response were mitigated by applying appropriate phase shifts for each individual layer. Measured results compared well with model predictions for all three cases, indicating that the transducer layering scheme was properly implemented. [This work was funded by NAVSEA NUWC Code 82 and Code 21.]

\section{9:45}

3aEA4. Structure-borne noise reduction for an infinite cylindrical shell (theory of elasticity). Sunghwan Ko, Woojae Seong, and Sangwoo Pyo (Dept. of Naval Architecture and Ocean Eng., College of Eng., Seoul National Univ., Seoul 151-742, South Korea)

A theoretical model was developed to evaluate the reduction of structure-borne noise generated by an axially symmetric ring force which is applied on the interior of the cylindrical shell. The vibrating cylindrical shell is coated with a microvoided elastomer that is acousically soft material designed for the reduction of the generated noise. The analytical model is a two-layer shell structure comprised of an outer layer of coating that is perfectly bonded to a cylindrical shell. The outer surface of the coating and the inner surface of the shell are in contact with water and air, respectively. The analysis for this problem is based on the theory of elasticity and pertinent boundary conditions. Effects of various parameters such as coating thickness and material properties on the noise reductions are presented.

\section{0:00-10:15 Break}

\section{0:15}

3aEA5. An optimization process for estimating two-port parameters. M. R. Serbyn and A. P. Kumar (Phys. Dept., Morgan State Univ., 1700 E. Cold Spring Ln., Baltimore, MD 21251, rserbyn@morgan.edu)

One process of parameter identification for a linear time-invariant two port consists of solving the inverse problem, given a set of values of the external variables. Such parameter estimates are subject to two sources of error: the measurement uncertainty and the propagation of error. The latter, in the worst case, can greatly exceed the former [M. R. Serbyn, J. Acoust. Soc. Am. 106, 2232 (1999)], but can be reduced by a judicious choice of the external variables, as implied by previously reported results, for example, [Li-Feng Ge, J. Acoust. Soc. Am. 97, 324-330 (1995)]. For this investigation a simple electrical circuit, characterized by a Moebius transformation, $w=(a 11 z+a 12) /(a 21 z+a 22)$, with known parameter values, was chosen. It provided a baseline for comparing the parameter values estimated from measurements of $z$ and $w$ made at the output and input ports, respectively. Each estimate was based on a set of three $(z, w)$ values and the reciprocity condition. By varying the nature and magnitude of the load impedance, $z$, and the corresponding input impedance, $w$, it was possible to minimize the errors in the computed values of the transformation matrix. Both simulated and experimental results were compared with the theoretical standard. 
10:30

3aEA6. An acoustic volumetric array system. Jonathan M. Rigelsford and Alan Tennant (School of Eng., Univ. of Hull, Hull HU6 7RX, UK, J.M.Rigelsford@eng.hull.ac.uk)

A microphone array system based on a three-dimensional random geometry is introduced. Three-dimensional microwave volumetric array antennas have recently been investigated as potential systems for radar and communications applications [A. Tennant and A. F. Fray, "A 64 element broad band volumetric array antenna," IEEE International Antenna and Propagation Symposium, Atlanta, 1998]. In a volumetric array, the elemental receivers occupy a solid region of three-dimensional space. A spherical array geometry is preferred. This provides the potential for full scan coverage. The projected aperture of a spherical array is direction independent, with the beam width and gain of the directional response being constant for all scan angles. The system developed consists of 64 omnidirectional microphones arranged at pseudorandom locations within a spherical volume. The nonperiodic element arrangement has two advantages. First, it eliminates the possibility of large grating lobes, and therefore enables wide-angle beam steering over a very large frequency bandwidth. Second, this geometry results in an array reception pattern with an average side-lobe level that is inversely proportional to the number of array elements. The potential of acoustic volumetric arrays is examined and the results of theoretical and experimental investigations presented. Applications areas include covert surveillance, imaging, and auditorium characterization.

\section{0:45}

3aEA7. Combined sound-pressure and pressure-gradient hydrophone design. Boris Aronov, Lawrence Reinhart, and David A. Brown (Acoust. Res. Lab., Dept. of Elec. and Comput. Eng. and the Ctr. for Marine Sci. and Tech., Univ. of Massachusetts, North Dartmouth, MA 02747)

A common design of a sound-pressure hydrophone relies on two bimorph piezoelectric circular plates symmetrically attached to a common base in the form of a ring or cylinder. This may be considered as having two inertially coupled partial systems vibrating in symmetrical and antisymmetrical modes. The symmetrical mode corresponds to the in-phase electrical connection of plates (hydrophone) and provides an output proportional to the omnidirectional sound pressure. The antisymmetrical mode corresponds to antiphase electrical connection of the plates and provides the figure-eight output proportional to the gradient of the pressure field. In order to make the electromechanical frequency responses of partial systems identical, the coupling between these systems must be made as small as possible. This can be achieved by proper design of the common base and achieving immunity to unwanted actions. The soundpressure hydrophone should be insensitive to acceleration caused by the pressure gradient and the pressure-gradient hydrophone should be insensitive to sound pressure. The properties of a combined hydrophone and the procedure of plate equalizing are investigated. The experimental data obtained are in a good agreement with theoretical expectations.

\section{1:00}

3aEA8. Review of ultrasonic transducer calibration techniques at Penn State University Applied Research Laboratory Aaron M. Foulk, W. Jack Hughes, and David Van Tol (Appl. Res. Lab., Penn State Univ., State College, PA 16804, aaronfoulk@ hotmail.com)

Recent emphasis on development of higher-frequency transducers for use in medical ultrasound and sonar applications has necessitated the creation of a reliable means of evaluation of these transducers. This work provides an overview of recent progress in the development of a facility for this purpose at the Pennsylvania State University Applied Research Laboratory. [Research funded by ONR.]

THURSDAY MORNING, 1 JUNE 2000

PEACHTREE BALLROOM, 8:30 A.M. TO 12:05 P.M.

\title{
Session 3aED
}

\section{Education in Acoustics: Publishing Excellence in JASA}

\author{
Allan D. Pierce, Cochair \\ Acoustical Society of America, P.O. Box 323, East Sandwich, Massachusetts 02537 \\ P. K. Raju, Cochair \\ Department of Mechanical Engineering, Auburn University, Ross 201, Auburn, Alabama 36849-5341
}

Chair's Introduction-8:30

Invited Papers

8:35

3aED1. Asking the right questions and following through: Obstacles encountered by prospective authors. Allan D. Pierce (Acoustical Society of America, P.O. Box 323, East Sandwich, MA 02537, adp@bu.edu)

Much of the best work in acoustics is presented at the Society's meetings. The Journal benefits if more presenters of such work submit archival-quality manuscripts. Obstacles include writer's block, counter-pressures from employers and others, inadequate support services, poor time organization, distrust (not necessarily unjustified) of the fairness and value of the JASA peer reviewing process, and a lack of appreciation of the long-term value of JASA publication. The Editor advocates expending the effort, gives suggestions on how authors can overcome the obstacles, and presents ideas (and pleas for suggestions from authors) as to how manuscript processing can be improved. The continued increase in quality of those papers eventually published remains the Journal's 
foremost priority, and authors can help by giving more attention to the selection of questions addressed in their research and by justifying the significance of such questions in their manuscripts. Recognition of the "right question" is an art that can be acquired, but which requires continued effort. Stimulation comes from many sources; the Editor especially recommends extensive reading of current literature, careful listening to talks at the "right" meetings, and a rapport with researchers who have a knack for asking the "right questions.",

\section{9:05}

3aED2. Frequent criticisms of submitted manuscripts. Sid P. Bacon (Dept. of Speech and Hearing Sci., Arizona State Univ., Box 871908, Tempe, AZ 85287-1908, spb@asu.edu)

Few manuscripts submitted to the Journal for consideration sail through without some critical comments by the reviewers and/or the associate editor. The manuscripts that the present editor sees are in the area of psychological acoustics, and many of the types of criticisms are undoubtedly similar to those made for manuscripts in other areas. A brief list (with sanitized examples) is given of the more common types of criticisms, and a discussion is given as to which of these lead to rejection of the article and as to which can be overcome by a revision. It is advised that authors view criticisms dispassionately and try to understand the substance of each criticism. In some cases, the criticisms are unjustified and the authors should endeavor to give a persuasive rebuttal rather than merely placating the reviewers. A good strategy for a successful author who desires rapid publication is to anticipate possible criticisms and to write the paper so that they will be avoided.

9:35

3aED3. Journal issues of peer review, contribution, and quality. William M. Carey (Dept. of Aerosp. and Mech. Eng., College of Eng., Boston Univ., 110 Cummington St., Boston, MA 02215, wcarey@bu.edu)

Allan Pierce, Editor-in-Chief, has recently written concise overviews of the Journal's history, publication criteria, and issues. To stimulate discussion, this paper follows his lead and addresses several resolvable issues that could improve the quality of published articles. Concerning the peer review and the reviewer, when should reviewers recuse themselves or disclose to the editor a close or adverse relationship with the author? What is the advisory role of the reviewer? What review criteria are necessary to ensure a timely, thorough, and fair review? Since articles are intended to be contributions to the field of knowledge, what constitutes a scholarly review of past work and faithful referencing? Should formal dissertations, laboratory reports, and edited conference proceedings be referenced if available from national information services or libraries? Should articles published in edited proceedings or formally archived reports be published as journal articles? Should research, numerical or experimental, be reported in incremental steps or as a completed investigation with significant results? Is the conduct of a large experiment sufficient for publication? Could prompt publication of letters to the editor about articles be used as a peer-feedback-quality control?

\section{0:05}

3aED4. The experience of an Associate Editor with the review process of papers in the Journal. Courtney B. Burroughs (Appl. Res. Lab., The Penn State Univ., P.O. Box 30, State College, PA 16804)

One of the most important parts of the Society is the Journal, in which information on new research findings are disseminated. Before a paper is published in the Journal, it must be approved by an Associate Editor who typically seeks the advice of two experts on the subject of the paper. Based on 6 years experience as an Associate Editor for the Journal, an overview of several aspects involved in the review process will be given. What constitutes a good review and what reviewers are looking for in an acceptable paper will be presented. Although the decision of acceptance rests with the Associate Editor, it is rare that this Associate Editor does not adhere to the recommendations made by reviewers. However, this Associate Editor does read all of the papers prior to deciding either to accept a paper after revisions are made in response to reviewer comments, reject the paper, or defer a final decision until after the paper has been revised and submitted for further review. An overview of this Associate Editor's experience with making the acceptance/rejection decision and what is felt to be the ingredients required for an acceptable Journal paper will be given.

\section{0:35}

3aED5. Anatomy of a readable publication. John C. Burgess (Dept. of Mech. Eng., Univ. of Hawaii, 2540 Dole St., Honolulu, HI 96822)

Richard Borden, some time ago, came up with a four-point formula that applies to any type of communication. The colloquial expressing of this formula consists of the phrases (1) Ho hum! (2) Why bring that up? (3) For instance! (4) So what? The present paper rephrases this formula so that it can be used by authors of research articles for JASA. The phrase "Ho hum," for example, translates to the requirement that the opening paragraph of an article should attract the reader's interest and motivate the reader to continue reading. Examples are taken from various JASA papers that indicate that those papers which are manifestly readable are in actuality (even if unconsciously) adhering to Borden's formula. It is suggested that authors who want their papers to be read and understood give serious consideration to making use of Borden's formula.

\section{1:05}

3aED6. The importance of critical book reviews. Philip L. Marston (Dept. of Phys., Washington State Univ., Pullman, WA 99164-2814, marston@wsu.edu)

According to C. S. Lewis, "Literature exists to teach what is useful, to honor what deserves honor, to appreciate what is delightful"' [C. S. Lewis, The Discarded Image (Cambridge U.P., Cambridge, 1967), p. 214]. While Lewis's context was remote from scientific and technical literature, these considerations are relevant to publication of timely book reviews in the Journal of the Acoustical Society of America. In addition to promoting a general awareness of books and the subject matter considered therein, book 
reviews can recognize and promote quality and the archiving of appropriate materials in libraries. The recognition of the real or apparent faults of a book is a valuable service along with the recognition of a book's positive attributes; however, it is appropriate to remember that aspects of the publication process may not be controlled by a book's author or authors.

\title{
11:35
}

3aED7. Publishing speech papers in JASA. Anders Lofqvist (Dept. of Logoped. and Phoniatrics, University Hospital, SE-221 85 Lund, Sweden, Anders.Lofqvist@logopedi.lu.se)

There are three sections for speech communication papers in JASA: production, perception, and processing and communication systems. In contrast to some other branches of acoustics, authors of speech-related papers have a wide variety of journals to choose from, covering several different disciplines. Among these are journals in psychology, cognitive science, linguistics, phonetics, speech and hearing science, otorhinolaryngology, neurophysiology, human movement science, and signal processing. Speech papers also cover production and perception in different human languages. In addition, papers in speech deal with both normal and clinical aspects, e.g., voice and speech disorders, and the effects of hearing impairment on speech. Papers in speech production are also concerned with analyzing articulatory movements as well as the mathematical modeling of phonation and articulation. The presentation will focus on why JASA should be a top choice for authors of papers in speech communication. Criteria for the selection of speech papers will be discussed as well as reviewing policy.

\section{Session 3aMU}

\section{Musical Acoustics: Visualization Methods for Musical Instrument Acoustics}

\author{
Uwe J. Hansen, Chair \\ Department of Physics, Indiana State University, Terre Haute, Indiana 47809 \\ Chair's Introduction-8:30 \\ Invited Papers
}

$8: 35$

3aMU1. Holographic analysis of musical instruments. Thomas D. Rossing (Phys. Dept., Northern Illinois Univ., DeKalb, IL 60115)

Musical instruments, when excited by bowing, plucking, blowing, or striking, vibrate in rather complicated ways. These complicated vibrations can be described in terms of normal modes of vibration, and understanding the normal modes or eigenmodes is important to understanding sound production in the instrument. Holographic interferometry offers by far the best spatial resolution of operating deflection shapes (and hence of normal modes), since it looks at an almost infinite number of points. Holographic interferograms may be recorded on photographic film or electronically by means of TV holography. We discuss various methods for holographic interferometry and its applications to several different types of musical instruments.

9:00

3aMU2. Extending modal analysis techniques: Representing the sound field. Uwe J. Hansen (Dept. of Phys., Indiana State Univ., Terre Haute, IN 47809) and Ingolf Bork (Physikalisch-Technische Bundesanstalt, Braunschweig, Germany)

Modal analysis enables a visual representation of structural normal-mode vibrations. This is accomplished by obtaining a series of transfer functions over a predetermined point grid on the structure, and fitting them to a model of coupled harmonic oscillators. These data can subsequently be visualized in slow-motion animation on the computer screen, representing the motion of the structure at the normal-mode frequencies. The key element in this procedure is the phase coherence between the excitation and the response signal. The technique can be extended to represent a sound field by retaining the phase coherence between a drive signal and a response signal detected by a microphone placed successively at points on a spatial grid in the sound field. The moving displacement on the computer screen represents pressure variations in their phase relation to the source oscillations. The technique will be illustrated with measurements in the air column of a flute, and the sound field above and below the sound board of a grand piano, as well as in a plane in front of the instrument.

9:25

3aMU3. Visualization of violin sound-radiation results from multiplanar near-field acoustic holography. Lily M. Wang and Courtney B. Burroughs (Grad. Prog. in Acoust., Penn State Univ., P.O. Box 30, State College, PA 16804)

The application of multiplanar near-field acoustic holography (NAH) to violins has produced an enormous amount of radiated sound-pressure and intensity data in three dimensions surrounding the instruments. The data have been analyzed to identify areas of significant sound energy radiation on the violin surface and the subsequent paths of that energy. The source regions have then been correlated to modal analysis results, as well as compared between structurally different violins. To complete such analyses has 
required powerful visualization tools. This presentation reviews the visualization software called application visualization system (AVS) and the techniques used for the investigation, including animating results on several sequential planes and viewing data as three-dimensional bricks that may be cut to view the interior behavior. [Work supported by NSF Graduate Research Fellowship, Lucent Technologies/Bell Laboratories GRPW Grant, and AAUW Selected Professions Dissertation Fellowship.]

9:50

3aMU4. The use of color in representing complex number data. Gabriel Weinreich (Phys. Dept., Randall Lab., Univ. of Michigan, Ann Arbor, MI 48109-1120)

With the general availability of color printers, the application of color for representing physical quantities has taken on a new interest. Since hue is by its nature a cyclic variable, it is ideally suited for representing the phase of a complex number; at the same time, color saturation can be used to specify amplitude. In this way, a complex number is conveniently represented by a single color; and a complex number that depends on, say, frequency, such as the input admittance of a wind instrument or the radiativity of a violin, can be mapped into a one-dimensional colored band. The great advantage of such a method is that these one-dimensional bands can be "stacked" in a perpendicular direction to indicate how the whole function behaves when another independent variable is introduced; for example, one might exhibit a radiativity as a function of both frequency and direction, or an input impedance as a function of both frequency and the number of open tone holes. A detailed application of the method to the "Swiss Cheese Violin" of Carleen Hutchins will be presented. [Work supported by NSF.]

10:15

3aMU5. Acoustic vortex appearing at the pre-steady state and disappearing at the steady state in organ pipes. Shigeru Yoshikawa (Dept. of Acoust. Design, Kyushu Inst. of Design, 4-9-1 Shiobara, Minami-ku, Fukuoka, 815-8540 Japan, shig@kyushu-id.ac.jp)

In visualizing the motion of the smoked jet during the attack transient, an acoustic (acoustically induced) vortex was observed alternately above and beneath the pipe edge at the pre-steady state just prior to the steady state. Interestingly enough, this acoustic vortex disappeared at the steady state. This finding is contrary to Fabre's, in which the acoustic vortex appears at the steady state [Acust. Acta Acust. 82, 863-877 (1996)]. The rotation of the acoustic vortex is the reverse of that of the hydrodynamic vortex produced at the initial phase of the attack transient. According to Howe's theory [J. Sound Vib. 70, 407-411 (1980)], our acoustic vortex seems to absorb the final excess in acoustic energy generation occurring at the pre-steady state and to lead the finally saturated amplification of the jet stability wave. The acoustic vortex may then be convected by the jet flow into regions where the vorticity can no longer continue to interact with the acoustic field. On the other hand, Fabre's acoustic vortex was interpreted as some sound source corresponding to higher harmonics. Slow-motion pictures captured with a high-speed digital video camera will be demonstrated.

10:40

3aMU6. Alternative mouthpiece design for viewing the lip reed in motion. R. Dean Ayers and Michael S. Lodin (Dept. of Phys. and Astron., California State Univ., Long Beach, 1250 Bellflower Blvd., Long Beach, CA 90840, rdayers@csulb.edu)

Mouthpieces for stroboscopic viewing of the lip reed have been designed for the full range of brass instruments, from trumpet to tuba. In order to provide an unobstructed frontal view of the lips, the axis of the backbore has been rotated $90^{\circ}$ from the axis of the rim, so that it emerges from the side of the cup. This is similar to the original design of Daniel W. Martin [J. Acoust. Soc. Am. 13, 305-308 (1942)]. Flat end windows are desirable for avoiding optical distortion, but they limit the range of viewing angles. Placement of the lips on the rim and the angle of the air channel between the lips can vary noticeably from performer to performer, so a flat window that works well for one embouchure may be quite inadequate for another. The large cup volumes of the low brass mouthpieces allow for greater flexibility in design without getting into severe distortion. A cylindrical end window with its axis parallel to that of the rotated backbore provides a wide range of viewing angles. A preliminary survey of embouchures used by beginning and advanced trombone players will be presented. [Work supported in part by the Scholarly and Creative Activities Committee at CSULB.]

\section{1:05}

3aMU7. A real-time/non-real-time spectrum analyzer for musical sounds. James W. Beauchamp and Timothy J. Madden (School of Music and Dept. of Elec. and Computer Eng., Univ. of Illinois at Urbana-Champaign, Urbana, IL 61801)

A color spectrum analyzer specialized for visualization of musical sounds has been developed for the Macintosh Power PC computer. Input samples (monaural or stereo) can be obtained from microphone or CD-ROM input in real time or from a sound file in nonreal time. Spectral analysis can be tuned specifically to the fundamental frequency of the input signal using sample rate conversion (tuned case), or it can be based on a specified window size at the input sample rate (nontuned case). In the tuned case each spectral component corresponds to a harmonic of the input. Several different displays can occur simultaneously: an oscilloscope waveform display; a 1D spectrum, where an amplitude-vs-frequency graph varies as time progresses; a spectral waterfall display; a 2D spectrum showing frequency vs time with amplitude registered as degree of display brightness; a 3D display showing amplitude vs time vs frequency at an arbitrary orientation. In addition to time-varying graphs which are available for both real and nonreal time, static plots are available for preanalyzed file input. For stereo signals it is possible to plot output/input transfer functions. Spectral time averaging is also available for cases where the instantaneous spectrum is unstable. 


\title{
Session 3aPAa
}

\section{Physical Acoustics: Acoustics of Multiphase Flow I}

\author{
Andrea Prosperetti, Cochair \\ Mechanical Engineering Department, Johns Hopkins University, 119 Latrobe Hall, 3400 North Charles Street, \\ Baltimore, Maryland 21218 \\ Lev A. Ostrovsky, Cochair \\ University of Colorado, NOAA/ETL, ET1, 325 Broadway, Boulder, Colorado 80303
}

Chair's Introduction-8:00

Invited Papers

8:05

3aPAa1. Acoustics of two-phase fluids. Robert I. Nigmatulin (Ufa Branch of Russian Acad. of Sci., 6 K. Marx Str., Ufa 450025, Russia)

Wave propagation in two-phase fluids is accompanied by a great variety of inter- and intraphase hydromechanical and thermophysical processes. These processes are initiated by a wave and, in turn, influence wave propagation strongly. Specific and anomalous features of the waves are the following: (1) Relaxation nonequilibrium processes need some relaxation time and space to transfer one equilibrium state in front of the wave to another equilibrium state behind. That is why the thickness of the wave or its relaxation zone may be large. (2) Attenuation of the wave is governed by nonlinear dispersion of disturbances and by dissipation because of the viscous friction between the carrier and dispersed phases and thermal dissipation, which is strongly dependent on phase transition possibility. (3) Bubbly liquid may show not only the attenuation, but even amplification of the shock wave. (4) For gas-drop mixtures with two-component carrier gas (noncondensable gas and vapor) it is possible to see an anomalous nonmonotonous effect of the mass drop content on the attenuation of the harmonic forced oscillation wave. (5) The breakdown of drops and bubbles has a strong influence on the wave propagation. It intensifies the interface force, heat, and mass transfer interactions. Sometimes it may appear as a "vapor explosion."

8:35

3aPAa2. The Nakoryakov-Pokusaev contribution to the acoustics of multiphase flow. Isaac Schreiber (Dept. of Chemical Eng., Ben-Gurion Univ. of the Negev, Beer-Sheva 84105, Israel)

Under the guidance of academician Nakoryakov and Professor Pokusaev, a cycle of theoretical and experimental studies of wave propagation in the gas-vapor bubbly liquid has been accomplished. It established the basis for the gas dynamics of gas-liquid multiphase flow. Here the main achievements will be listed. (1) The bridge was built between the modern theory of nonlinear waves and the progress in heat transfer. (2) The comprehensive picture of the dynamics and structure of the wave propagation in gas-vapor bubbly liquid was found. The main bright results are the following: discovery of oscillating and monotonic shock waves and derivation of criteria for their existence; models for sound propagation in bubbly liquid and in boiling water (Korteveg-Burgers and Klein-Gordon approximations); acoustics of the slug-plug regime; two-wave structure of shock waves with shape fronts. These results strongly influenced the modern theory of the acoustics of foams and solids saturated by bubbly liquid. This lecture is dedicated to the jubilees of Nakoryakov (65th birthday) and Pokusaev (65th birthday).

9:05

3aPAa3. Nonlinear wave interactions in water with bubbles: Solved and unsolved problems. Lev A. Ostrovsky (Univ. of Colorado, CIRES/NOAA ETL, 325 Broadway, R/E/ET1, Boulder, CO 80303)

This lecture is an outline of some nontrivial aspects of nonlinear acoustics of bubbly liquids. It is common knowledge now that a very small volume content of bubbles can increase the nonlinearity parameter of the gas-liquid mixture by several orders. Basic problems of nonlinear wave propagation in such media with shock wave formation, harmonic generation, etc., have been thoroughly discussed during the last decades, especially for a less realistic case of equal-size bubbles. Not as well understood are waves in bubbles widely distributed in sizes, especially nonlinear scattering problems when the standard "continual" approximation is inapplicable. Also, collective behavior of bubbles interacting with each other (not just via the macroscopic acoustic field) can radically change the nonlinear properties on a medium. Here, some relevant theoretical and experimental results are discussed, including nonlinear scattering of sound on sea bubbles; the use of resonance bubble layers to enhance nonlinear transformation; and some effects of bubble interaction due to period-averaged forces such as radiation pressure. The relationship between coherent and incoherent parts of acoustic field and some ideas of an "acoustic masker" are also discussed. 
3aPAa4. The coupling of airborne sound into air-filled poro-elastic soils. James M. Sabatier (Natl. Ctr. for Physical Acoust., Univ. of Mississippi, University, MS 38677, sabatier@olemiss.edu)

The coupling of airborne sound into near-surface soils will be reviewed. Particular emphasis will be given to research conducted at the National Center for Physical Acoustics over the past 10 years, including the works of Arnott, Frederickson, and Hickey. For the purpose of acoustically describing pore properties, the ground is modeled as an air-filled, porous, rigid-framed material. Measurements using both transmission and reflection of sound have been used in conjunction with rigid framed acoustic propagation models to determine air flow resistivity, porosity, and tortuosity. In other research, the ground is modeled as a layered poro-elastic medium following the work of Biot. Measurements of the motion of the elastic frame are made using geophones, buried microphones, and laser Doppler vibrometers. Responses of these sensors to airborne or acoustically induced seismic waves will be described. A recent application of this understanding of the ground is the detection of buried land mines. Some brief comments will be made with regard to this application.

\section{0:00-10:20 Break}

10:20

3aPAa5. Forward and inverse modeling in porous media. Joseph F. Lingevitch, Michael D. Collins (Naval Res. Lab., Washington, DC 20375), Andrew J. Fredricks, and William L. Siegmann (Rensselaer Polytechnic Inst., Troy, NY 12180)

Techniques for solving forward and inverse wave propagation problems involving poroelastic layers will be discussed. Parabolic equation techniques are efficient for solving problems in laterally varying media. A parabolic equation for poroelastic media has been developed and applied to problems in ocean acoustics [J. Acoust. Soc. Am. 98, 1645-1656 (1995)]. This approach has been generalized to the anisotropic case. The smallness of shear speeds in many ocean sediments has motivated the study of poroacoustic media [J. Acoust. Soc. Am. 104, 783-790 (1998)], which is a limiting case of Biot theory in which the rigidity vanishes. Although parabolic equation techniques have not been fully generalized from acoustics to poroelasticity, studying the intermediate case of poroacoustics has helped to bridge the gap. The parabolic equation techniques have been used as tools for solving inverse problems. This approach is presently being applied to field data. One of the issues that arises in solving the inverse problem is the mapping between the coefficients of the wave equation and the wave speeds. The inverse of this mapping can be used to define problems in terms of natural parameters rather than moduli. [Work supported by ONR.]

\section{0:45}

3aPAa6. Methods for measurement of the attenuation coefficient and phase velocity of Optison and their relevance to in vivo efficacy. Michael S. Hughes, Alexander L. Klibanov, John H. Wible, and Gary H. Brandenburger (Mallinckrodt, Inc., 675 McDonnel Blvd., Hazelwood, MO 63042, mxhughe@mkg.com)

We report methods and results of measurements of contrast media attenuation coefficient and phase velocity for concentrations ranging from $1.7 \times 10^{5}$ to $21 \times 10^{6}$ particles $/ \mathrm{ml}$. These concentrations cover the range likely to occur during in vivo application of ultrasound contrast media such as Optison. For the concentrations cited in this study, the attenuation coefficient exhibits a single peak between 1 to $2 \mathrm{MHz}$, while the phase velocity rises sharply and then levels off, typically near the same frequency at which the attenuation peak occurs. Previous studies from our laboratory have shown that these acoustic properties of ultrasound contrast media can change significantly with changing experimental conditions. For instance, the frequency at which the peak attenuation of Albunex occurs can shift by as much as 1 to $1.5 \mathrm{MHz}$ as the measurement temperature is increased from $22^{\circ}$ to $37{ }^{\circ} \mathrm{C}$. Similarly, the attenuation peak width (full width at half maximum) can narrow by more than a factor of 2 (the exact amount depends on concentration). The peak attenuation values can shift by as much as $50 \%$ with increasing acoustic pressure. The primary aim of this study is to measure acoustic properties which are as close as possible to those occurring in clinical practice.

\section{Contributed Papers}

\section{1:10}

3aPAa7. Design and characterization of a system for acoustophoresis. Todd L. Brooks and Robert E. Apfel (Dept. of Mech. Eng., Yale Univ., New Haven, CT 06520-8286, todd.brooks@yale.edu)

The manipulation of particles using acoustic forces is a basis for a potentially valuable continuous separation method. The method is unique in that particles can be distinguished based on their relative compressibilities, as well as on mass density and size. An acoustic standing wave is oriented perpendicular to the fluid flow direction which enables an incoming particle mixture to be separated into different streams at the fluid output. Effects which cause mixing such as heat convection and acoustic streaming must be minimized through careful design. An imaging system has been implemented to record the trajectories of individual particles as they flow through the separation cell. Trajectories of various types of particles ranging from 5 to 55 microns are compared to theoretical esti- mates of separation effectiveness, with a particular emphasis on the role of secondary inter-particle acoustic forces. [Work supported by NASA through Grant No. NAG8-1351.]

\section{$11: 25$}

3aPAa8. Just a box of rain: Making rain with sound. Gretchen Snoeyenbos, Giovanni De Santi, and R. Glynn Holt (Dept. of Aerosp. and Mech. Eng., Boston Univ., 110 Cummington St., Boston, MA 02215)

Clouds don't always produce rain. One of the reasons is that microdroplet coalescence does not occur fast enough to produce drops large enough to fall. A brief review of the literature on particle and aerosol agglomeration will be presented. The results of an experiment in which ultrasonic standing waves are employed to enhance microdroplet coalescence in a laboratory cloud will be reported. A force analysis will be 
presented to allow prediction of the efficiency of the coalescence process for arbitrary acoustic field frequency and droplet radius. If possible, a comparison will be made between experimental results and model predictions.

\section{1:40}

3aPAa9. New models and measurements concerning sound attenuation in concentrated airborne suspensions. Keith Attenborough, Qiang Wang (Univ. of Hull, Hull HU6 7RX, UK), and Steve Woodhead (Univ. of Greenwich, Chatham Maritime ME4 4AW, UK)

In the inertial regime of frequency-radius space, irregularity and aggregation of particles can result in values of acoustic attenuation that are significantly different from those predicted by assuming separated smooth spherical particles. Data obtained previously from suspensions of alumina particles and olivine sand in air at audio frequencies, together with new data obtained at low ultrasonic frequencies in suspensions of glass beads and silica flour, are compared with predictions. It is shown that neither a coupled-phase theory modified to allow for nonspherical shapes nor effective radius theories are able to account for these data. Qian [Phys. Rev. E 53(3), 2304-2306 (1996)] has suggested that suspensions may be treated as fractal media and used the acoustic Reynolds number as the fractal dimension in modifying scattering theory. A new fractal modification of multiple scattering theory for acoustic attenuation is derived. The theory uses $\omega \tau_{v}$ ( $\omega$ is the angular acoustic frequency, $\tau_{v}$ is the dynamic relaxation time of the particles) as a fractal scale. Fitted values of fractal dimension obtained at a single frequency are found to enable fits with data at other frequencies. Moreover, the fractal approach is found to enable discrimination between the effects of particle irregularity and aggregation. [Work supported by EPSRC (UK).]

THURSDAY MORNING, 1 JUNE 2000

PEACH ROOM, 8:15 A.M. TO 12:00 NOON

\title{
Session 3aPAb
}

\section{Physical Acoustics: Scattering and Radiation}

\author{
Christine Valle, Chair \\ Mechanical Engineering Department, University of Maine, Orono, Maine 04473
}

\section{Contributed Papers}

$8: 15$

\begin{abstract}
3aPAb1. Amplitude variation of resonant frequencies and Q-factor in impulse acoustic resonant spectroscopy. Alexander M. Sutin (Stevens Inst. of Technol., 711 Hudson St., Hoboken, NJ 07030, asutin@stevens-tech.edu), Robert A. Guyer (Univ. of Massachusetts, Amherst, MA 01003), and Paul A. Johnson (Los Alamos Natl. Lab., Los Alamos, NM 87545)
\end{abstract}

One of the oldest and simplest nondestructive tests of the quality of an object is to tap it and listen to the radiated sound. The translation of this test into a modern instrumentation system leads to impulse acoustic resonance spectroscopy, IARS. The processing of the acoustic time train after impulse excitation (tap) allows one to follow $\mathrm{Q}$ and resonance frequencies as a function of time. Examination of the time train can reveal important properties of this elastic state and of the state of the object. IARS will be illustrated with data on a suite of automobile brake drums made of powdered aluminum. The time evolution of $\mathrm{Q}$ and a resonance frequency will be described. Typically $\mathrm{Q}$ is of order one-half of its late time (equilibrium) value at short times whereas a resonance frequency shifts by of order $0.2 \%$ from early $(0.2 \mathrm{~s})$ to late $(5 \mathrm{~s})$ time. These qualitative properties have two possible explanations: (1) amplitude-dependent internal friction or (2) the "slow dynamics" that is characteristic of elastic systems inhabited by hysteretic elastic elements. [Work supported by Stevens and by the Department of Energy: Office of Basic Energy Sciences.]

\section{8:30}

3aPAb2. On a novel application of the Helmholtz integral in the development of a noiseless sonar. Anthony J. Romano, Joseph A. Bucaro, Brian H. Houston, and Earl G. Williams (Naval Res. Lab., Washington, DC 20375-5350)

A novel application of the Helmholtz integral is presented in the development of a noiseless sonar. Given total pressure and normal velocity information over the surface of a structure which has been excited both by an incident field as well as interior noise sources, when the field point resulting from the Helmholtz integral is evaluated inside the surface of the structure, only the incident field remains. Therefore, it is possible to integrate out the effects of both self-noise (due to interior noise sources) and scattering (due to incident excitation) throughout a "virtual" volumetric sensor array projected within the structure. This approach will be discussed and demonstrated using numerical results based on finite element simulations. [Work supported by ONR.]

\section{$8: 45$}

3aPAb3. Inverse scattering of three-dimensional obstacles. Dilip N. Ghosh-Roy (SFA, Inc., 1401 McCormick Dr., Landover, MD 20785), Luise S. Couchman, and Jeremy A. Warner (Naval Res. Lab., Washington, DC 20375-5350)

The results of three-dimensional reconstructions of obstacles in an infinite, homogeneous ambience from the far-field scattering of plane acoustic waves in a resonance frequency region are presented. The reconstructions include penetrable and impenetrable objects. Also discussed are reconstructions of orientations (that is, Euler angles), surfaces, and interiors of penetrable axisymmetric scatter. In addition, inverse obstacle scattering using a highly restricted angular aperture in monostatic and quasimonostatic data collection configurations is also discussed.

\section{9:00}

3aPAb4. Backscattering enhancements of ultrasound by tilted solid plastic cylinders in water due to the caustic merging transition: High angular resolution scans. Florian J. Blonigen and Philip L. Marston (Dept. of Phys., Washington State Univ., Pullman, WA 99164-2814, blonigen@physalpha.physics.wsu.edu)

Bulk shear and longitudinal waves can make important contributions to the scattering of ultrasound by tilted finite plastic and rubber cylinders in water when the phase velocity of the wave is less than the speed of sound of the surrounding fluid. At a certain critical tilt angle, a back- 
scattering enhancement is observed as a result of merging rainbow caustics associated with bulk transmitted rays internally reflected off the cylinder truncation [F. J. Blonigen and P. L. Marston, J. Acoust. Soc. Am. 107 (2000)]. The backscattering away from this angle is otherwise weak. The critical angle is calculated using the Bravais effective refractive index for the projections of rays on the base plane of the cylinder. New ultrasonic observations are reported for a polystyrene cylinder over a wide range of tilt angles at high angular resolution. The backscattering amplitude angle scan takes on the shape of a Pearcey function with the peak amplitude offset from the critical angle. Backscattering amplitudes at the critical angle are also compared with a ray theory prediction as a function of frequency for both the polystyrene and a silicone rubber cylinder. [Work supported by ONR.]

\section{9:15}

3aPAb5. Backscattering enhancements associated with mode conversion in thin tilted circular plates in water: Observations and Gaussian beam model. Brian T. Hefner and Philip L. Marston (Phys. Dept., Washington State Univ., Pullman, WA 99164, bhefner@mail.wsu.edu)

When a thin tilted circular plate is illuminated with high-frequency sound, a significant enhancement occurs when the angle of incidence corresponds to the extensional wave coupling angle. It has been found that there are two mechanisms responsible for this enhancement. The largest response is due to the extensional leaky wave which travels along the diameter and reflects from the plate edge. This can be modeled in the thin-plate limit using a Gaussian beam model which was first developed for the reflection of leaky Rayleigh waves on tilted truncated cylinders [K. Gipson and P. L. Marston, J. Acoust. Soc. Am. 107, 112-117 (2000)]. The second response is due to extensional waves traveling along off-diameter paths. When these waves strike the plate edge, they mode-convert into shear waves. These shear waves cross the diameter to strike the opposite edge and mode-convert into extensional waves which then reradiate back towards the source. Since the shear waves do not leak energy into the water, this enhancement has a relatively slow decay as the waves undergo multiple reflections around the plate edge. The Gaussian beam model has been extended to quantitatively model this enhancement in the thin-plate limit. [Work supported by ONR.]

\section{9:30}

3aPAb6. Raman-Ray whispering gallery experiment and enhanced backscattering from merged caustics: Optical analogies of acoustical wavefields. Philip L. Marston, Charles C. Barnes, Yibing B. Zhang, and David B. Thiessen (Dept. of Phys., Washington State Univ., Pullman, WA 99164-2814)

Two optical experiments for viewing wavefields associated with acoustical processes are demonstrated. The first is a modern version of a little-known whispering gallery experiment by Raman and his associate Ray [C. V. Raman and G. A. Sutherland, Proc. R. Soc. London, Ser. A 100, 424-428 (1922); B. Ray, Bull. Calcutta Math. Soc. 12, 225-230 (1922)], which simulate wavefields for a source on a curved reflecting surface. Our simulation uses a long, narrow front-surface mirror bent uniformly by attached weights. The light source is the diffraction of laser light by a knife edge placed adjacent to the mirror. The caustic associated with once-reflected rays (a cardioid curve) is easily distinguished as are other caustics associated with rays having only a few reflections. Amplitude contributions near each caustic are approximated by Airy functions. The second experiment is analogous to an acoustical backscattering enhancement by bluntly truncated tilted penetrable circular cylinders associ- ated with the merging of end-reflected rainbow caustics [F. J. Blonigen and P. L. Marston, J. Acoust. Soc. Am. (in press)]. Light backscattered by a tilted blunt glass fiber displays the merged caustics for the appropriate tilt. [Work supported by ONR and NSF-CRDC.]

\section{9:45}

3aPAb7. Numerical results for acoustic scattering from a coated wedge. R. Hughes, J. Niemiec, S. Solomon (Naval Surface Warfare Ctr., Carderock Div., Bethesda, MD 20817-5700), and Herbert Überall (Catholic Univ. of America, Washington, DC 20064)

Earlier work on the acoustic wedge problem mainly dealt with an impenetrable wedge, including the work of Sommerfeld (1896), Bromwrich (1915), Carslaw (1920), and Oberhettinger (1954, 1958). The present investigation assumes a line source parallel to the edge of the wedge, and extends Oberhettinger's approach for the scattering of a sound wave from the edge of a perfectly reflecting wedge, to the case that the field does not satisfy a Dirichlet or Neumann boundary condition on the wedge surface, but an impedance boundary condition. Such a condition is capable of describing the effect of a lossy coating on the wedge surface as shown for the electromagnetic case (Überall, 1964, 1966), and the corresponding impedance for the acoustic case has been obtained here for one, two, and multiple layers of coating. Numerical results for the wedgescattered sound intensity will be presented and discussed.

\section{0:00-10:15 Break}

\section{$10: 15$}

3aPAb8. Radiation impedance matrix of a rectangular aperture, with arbitrary vibration, in a rigid baffle. Allan D. Pierce and Robin O. Cleveland (Dept. of Aerosp. and Mech. Eng., Boston Univ., Boston, MA 02215, adp@bu.edu)

The present paper shows that multidimensional integrals associated with radiation from a rectangular surface in a plane rigid baffle can be reduced to one-dimensional integrals. If the vibrational excitation is expanded into a summation over a set of basis functions, the surface pressure and velocity may be related by an infinite matrix. Each element of the matrix is expressed as a quadruple integral. The numerical evaluation of such integrals, although possible, is prohibitively time consuming for many practical applications. However, for some choices of the basis functions, each quadruple integral may be reduced analytically to single integrals. Explicit expressions are given for square surfaces and with the basis functions taken as products (or sums of products) of trigonometric functions. Numerical results agree well with previous results published in the literature for the pressure distribution over a surface when the vibration is uniform.

10:30

3aPAb9. Dispersion of axially symmetric waves in fluid-filled cylindrical shells. X. L. Bao, H. Überall (Phys. Dept., Catholic Univ. of America, Washington, DC 20064), P. K. Raju, A. C. Ahyi (Auburn Univ., Auburn, AL 36849-5341), I. K. Bjørnø, and L. Bjørnø (Tech. Univ. of Denmark, DK-2800 Lyngby, Denmark)

Acoustic waves normally incident on an elastic cylindrical shell can cause the excitation of circumferential elastic waves on the shell. These shells may be empty and fluid immersed, or fluid filled in an ambient 
medium of air, or doubly fluid loaded inside and out. Circumferential waves on such shells have been investigated for the case of aluminum shells, and their phase-velocity dispersion curves have been obtained for double fluid loading [Bao, Raju, and Überall, J. Acoust. Soc. Am. 105, 2704 (1999)]. Similar results were obtained for empty or fluid-filled brass shells [Kumar, Acustica 27, 317 (1972)]. We have extended the work of Kumar to the case of fluid-filled aluminum shells and steel shells imbedded in air. These cases demonstrate the existence of circumferential waves traveling in the filler fluid, exhibiting a certain simplicity of the dispersion curves of these waves. This is in striking contrast to the results for double (outside and inside) loading by two fluids of comparable density, where circumferential waves in both external and internal fluids were found, their interaction causing segmentation and repulsion phenomena of their dispersion curves. The condition of standing circumferential waves determines the eigenfrequency spectrum of the shell.

\section{$10: 45$}

3aPAb10. A general acoustic background for evacuated and waterfilled submerged shells. Michael F. Werby (NRL Code 7181, Stennis Space Center, MS 39571, werby@nrlssc.navy.mil) and Herbert Uberall (Catholic Univ. of America, Washington, DC)

Historically, the acoustic background for elastic solids dates back to the early 1980 s to the work of Flax, Uberall, and Dragonette. Werby extended this concept in 1991 to evacuated elastic shells by employing the concept of entrained mass. Here we extend the concept to one valid for a water-filled shell. The new background is equally valid even for the limit of an evacuated shell. We derive the equations used in the method and illustrate its use for a variety of problems.

\section{1:00}

3aPAb11. A comparative study of the scattering cross sections from incident plane wave acoustic waves on submerged evacuated and water-filled elastic shells. Herbert Uberall (Dept. of Phys., Catholic Univ. of America, Washington, DC, huberall@aol.com) and Michael F. Werby (NRL, Stennis Space Center, MS)

Employing the acoustic background for the case of elastic solids and elastic shells has facilitated the study of elastic resonances excited by incident acoustic signals. Once an adequate acoustic background became available it became possible to properly isolate and characterize such resonances. The recent development of an acoustic background for water-filled elastic shells allows for the characterization of water-filled shells. We present a comparative study for the elastic shell case for the evacuated and water-filled cases and thereby seek to elucidate new resonance mechanisms for the fluid-filled case.

\section{1:15}

3aPAb12. Multiple scattering attenuation and anisotropy of ultrasonic surface waves. John A. Scales and Kasper Van Wijk (Ctr. for Wave Phenomena, Colorado School of Mines, Golden, CO 80401, kasper@dix.mines.edu)

Multiple scattering of waves induces bulk effects such as attenuation and anisotropy that are important in seismology, optics, medical imaging, and other fields involving propagation in disordered media. Measurements are reported of ultrasonic surface wave propagation in a strong-scattering, quasiperiodic medium consisting of a grooved surface of aluminum. Using noncontacting optical methods we have tracked the evolution of surface wave pulses within the scattering medium. Waves propagating parallel to the grooves propagate nearly attenuation and dispersion free, whereas waves propagating normal to the grooves are dispersed and exponentially attenuated with distance as energy is transferred from the direct pulse into the multiple-scattering coda. We measure this attenuation length and show that there is, in addition, a scattering induced anisotropy in the phase velocity. [This work was partially supported by the U.S. Army Research Office under Grant No. DAAG55-98-1-0070.]

\section{$11: 30$}

3aPAb13. Damage detection in thin composites using guided waves. Christine Valle (212 Boardman Hall, Univ. of Maine, Orono, ME 04473, valle@umeme.maine.edu)

Lightweight, high-temperature composite materials have the potential to provide significant economic and performance benefits to aerospace structures. However, one problem inherent in using composites within complex structures in harsh environments is the variety of damage mechanisms experienced by the composite itself. Therefore, conventional NDE techniques that are sensitive to only one kind of damage can give an overly optimistic assessment of the health of the composite. A global measure that takes into consideration all of the different damage mechanisms better addresses the nondestructive evaluation needs of composites used in critical applications. This study investigates the relationship between the composite's dispersion relationship_-generated with a $2 \mathrm{D}$ fast Fourier transform representation of transient ultrasonic signals, created with a finite-element (FE) model — and the energy content of the structure, as a function of damage amount. The total amount of energy contained in the structure is measured, and when normalized with respect to a virgin state, illustrates the loss due to a given amount of damage. This method provides a quantitative assessment of the amount of damage in the material, and establishes the numerical and analytical foundations for the application of FE models to damage monitoring in composites.

\section{1:45}

3aPAb14. New techniques for the characterization of materials in situ using ultrasound. Konstantinos Christidis and G. P. P. Gunarathne (Dept. of Electron. and Elec. Eng., The Robert Gordon Univ., Aberdeen AB11FR, UK)

Characterization of materials in situ using ultrasound is an attractive concept but has two main problems due to: (1) arbitrary geometrical shape of the target, and (2) surface roughness of the material. Some work has been previously reported in measuring the surface roughness using ultrasound [J. Stor-Pellinen and M. Luukkala, Sensors and Actuators A49, 37-40 (1995)] and also material characterization using ultrasonic feature extraction [G. P. P. Gunarathne and R. W. Keach, Ultrasonics 34, 411419 (1996)]. However, there is no integrated approach developed to characterize materials in situ, taking into account the effects due to shape and roughness of the target. In this work a comprehensive study of the effects of target shape and roughness on ultrasonic time- and frequency-domain measurements were carried out. A mathematical model which compensates for these variables was then developed, so that the compensated signals could be used to evaluate the material properties. Using these mathematical models and practical measurements, a formula which compensates for the combined effect of curvature and target roughness was developed. This model was then used to characterize materials in situ, using stored data and artificial neural networks. Close agreement between theoretical and practical measurements has been demonstrated. 


\title{
Session 3aPP
}

\section{Psychological and Physiological Acoustics: Localization and Precedence (Poster Session)}

\author{
Ruth Y. Litovsky, Chair \\ Hearing Research Center, Department of Biomedical Engineering, Boston University, 44 Cummington Street, \\ Boston, Massachusetts 02215
}

\section{Contributed Papers}

\begin{abstract}
All posters will be on display from 8:30 a.m. to 12:00 noon. To allow contributors an opportunity to see other posters, contributors of odd-numbered papers will be at their posters from 8:30 a.m. to 10:15 a.m. and contributors of even-numbered papers will be at their posters from 10:15 a.m. to 12:00 noon.
\end{abstract}

\begin{abstract}
3aPP1. Spatial unmasking of nearby pure-tone sources in a simulated anechoic environment. Norbert Kopčo (Dept. of Cognit. and Neural Systems, Boston Univ., Boston, MA 02215, kopco@cns.bu.edu) and Barbara G. Shinn-Cunningham (Boston Univ., Boston, MA 02215)
\end{abstract}

Spatial unmasking of nearby pure-tone sources masked by broadband noise was studied in a simulated anechoic environment. The predicted amount of binaural unmasking was calculated using interaural differences from a spherical-head model [B. G. Shinn-Cunningham, S. G. Santarelli, and N. Kopčo, J. Acoust. Soc. Am. (in press)] and the Colburn model of binaural processing [H. S. Colburn, J. Acoust. Soc. Am. 61, 525-533 (1977)]. Predictions were generated for several spatial configurations of signal and masker in the horizontal plane (varying target and masker azimuthal positions and distances) and for multiple tone frequencies. Predictions were compared with behaviorally measured detection thresholds obtained with headphone simulations using individually measured HRTFs. Results show that changes in detection threshold for free-field signals are dominated by monaural effects due to large interaural level differences. Overall, spatial unmasking effects are very large for nearby sources due to simple energy effects at the better ear. Binaural unmasking is observable only for a subset of spatial configurations where the energy of signal and/or noise at both ears is sufficient to allow binaural processing, and even for these configurations the effect is generally weaker than that observed with distant sources. [Work supported in part by AFOSR Grant F49620-98-1-0108.]

3aPP2. Spatial unmasking for nearby speech sources in a simulated anechoic environment. Jason Schickler (Hearing Res. Ctr., Dept. of Biomed. Eng., Boston Univ., Boston, MA 02215), Norbert Kopčo, Barbara G. Shinn-Cunningham, and Ruth Y. Litovsky (Boston Univ., Boston, MA 02215, shinn@cns.bu.edu)

The "cocktail party effect" is traditionally studied with sources varying in azimuth, where binaural and level cues are large. In these conditions, speech intelligibility improves dramatically when the target speech and maskers are spatially separated. In the present study, we investigated spatial unmasking of nearby sources for several configurations of target and sources varying in distance and azimuth. Targets were sentences from the IEEE corpus and maskers were speech-shaped noise. Locations were simulated over headphones using anechoic head-related transfer functions (both individually measured and using a spherical-head model). Speech reception thresholds were measured adaptively, varying target level while keeping masker level constant. For nearby sources, overall energy effects (due to changes in target and masker level with distance) are large. Therefore, target level was normalized to equate the signal-to-noise ratio at the better ear in all conditions. This approach allowed direct measurement of binaural unmasking in the various conditions. Overall energy effects (removed by the normalization) are also reported. Results are compared with detection threshold measurements from similar experiments. [Work supported in part by AFOSR Grant F49620-98-1-0108 to BGSC and NIDCD Grant DC02696 to RYL.]

3aPP3. Localization of ripple-spectrum noise. Ewan A. Macpherson (Kresge Hearing Res. Inst., Univ. of Michigan, Ann Arbor, MI 48109)

Ripple-spectrum stimuli were used to investigate the level of spectral detail important for use of spectral cues for vertical-plane localization. Free-field localization judgments were obtained for $250-\mathrm{ms}, 0.5-16 \mathrm{kHz}$ noise bursts with various log-ripple spectra. Ripple density was varied between 0.25 and 8 cycle/octave at a depth of $\pm 20 \mathrm{~dB}$. Depth was varied between \pm 5 and $\pm 20 \mathrm{~dB}$ at a density of 1 cycle/oct. Multiple ripple phases were employed at each combination of density and depth. \pm 20 -dB rippled spectra substantially increased errors in vertical-plane localization in the range $0.5-2$ cycle/oct. When systematic errors occurred, judgments were biased toward the location at which the listener's directional transfer function (DTF) best matched the rippled source spectrum filtered by the target-location DTF. At 1 cycle/oct, localization accuracy was degraded only for ripple depths at or above $\pm 15 \mathrm{~dB}$. Although the upper limit for ripple discrimination is 10 cycle/oct [Supin et al., J. Acoust. Soc. Am. 106, 2800-2804 (1999)], these results indicate that detail finer than 2 ripples/oct does not strongly influence spectral cue processing. This has significance for localization modeling and for virtual-stimulus synthesis. [Work supported by NIH Grants R01DC00420 and T32DC00011.]

3aPP4. Free-field and virtual studies of the precedence effect in the median-sagittal plane: Duration effects. Gerald Ng, Roberto Dizon, Ruth Litovsky, and H. Steven Colburn (Hearing Res. Ctr., Boston Univ., 44 Cummington St., Boston, MA 02215)

The study of precedence in the median-sagittal plane is made difficult by poor localization ability in this plane, as well as by front-back confusions. We report on both free-field and virtual localization studies in which 
a source and simulated reflection are presented from two speakers chosen from an array of six, all of which are in the frontal median-sagittal plane. Subjects perform a forced-choice identification of which speaker emitted the combined stimulus. Stimuli consisted of lead-lag pairs of 1-, 10-, 25-, or 50-ms broadband noise bursts, where the onset of the reflection lags that of the source by $2 \mathrm{~ms}$. Single-source cases were presented intermixed with the lead-lag paired stimuli. In the free-field condition, identification accuracy increased with duration for the single-source stimuli. For the precedence stimuli, a majority of responses is biased towards the elevation of the leading stimulus. However, details of individual performance show some evidence of response bias towards certain elevations, bimodal distributions, and perceptual averaging of lead and lag locations. Virtually presented stimuli using individualized HRTFs yielded localization judgments comparable to free-field stimuli for single-source conditions, but not for lead-lag pairs. [Work supported by NIH (DC02696 and DC00100) and ONR-MURI (Z883401).]

3aPP5. Investigations of the precedence effect with narrow-band continuous noise. Roberto Dizon and H. Steven Colburn (Hearing Res. Ctr. and Biomed. Eng. Dept., Boston Univ., Boston, MA 02215)

The precedence effect has been shown to be strong for stimuli with onset transients, e.g., clicks. Zurek [J. Acoust. Soc. Am. 67, 952-964 (1980)] showed that a continuous, broadband noise whose fine structure leads that of a simulated reflection dominates lateralization even when onset gating cues have been removed. We report on experiments similar to those of Zurek, but using narrow-band noises of varying bandwidths and center frequencies. In a 2IFC headphone task, the leading and lagging stimuli are placed on either side of midline $( \pm 300-\mu$ s ITD) in the first interval, and the signals to the two ears are switched in the second interval. Subjects report whether the stimulus in the second interval is to the right or left of the first. Results are consistent with a precedence effect for bandwidths as narrow as a critical band. For narrower bandwidths, onset dominance is still observed, but with oscillations in lateralization consistent with the interaural differences expected for narrow-band stimuli, given the ITDs of the lead and lag stimuli and the lead-lag delay. As bandwidths approach zero, lateralization is dominated by these interaural differences, and onset dominance is not evident. [Work supported by NIH (DC00100) and ONR-MURI (Z883401).]

3aPP6. Optimal integration of directional information across a population of simple MSO neurons. Sergei Lubensky (Dept. of Elec. Eng. and Computer Sci. Res. Lab. of Electron., MIT, 77 Massachusetts Ave., Cambridge, MA 02139), Barbara G. Shinn-Cunningham, H. Steven Colburn (Boston Univ., Boston, MA 02215), and Nathaniel I. Durlach (MIT, Cambridge, MA 02139)

A simple computational model of source laterality was developed that optimally combines information across a population of neurons in the medial superior olive. A simple point-neuron MSO model was used to simulate the output of each of the interaural-phase sensitive neurons in the population. Each neuron has a best frequency and best interaural phase delay (IPD), generating an intermediate display similar to that proposed by Colburn [J. Acoust. Soc. Am. 61, 525-533 (1977)]. Information in this population was combined (i.e., across best frequency and best IPD) to determine the a posteriori probability of a source from each possible angle relative to the interaural axis. From this output, source laterality was predicted from the maximum likelihood estimate of source direction. Similar to previous models [R. M. Stern and C. Trahiotis, Proceedings 11th International Symposium on Hearing (1997), pp. 336-345], this approach looks for consistency in the neural responses across frequency. However, the current model explicitly weights information corresponding to different best frequencies and IPDs based on the uncertainty in the responses of the neurons in the population. Lateralization predictions were generated for a variety of sources and compared with results from the literature. [Work supported in part by AFOSR Grant No. F49620-98-1-0108.]

3aPP7. Auditory search asymmetry with pure tone, narrow-band noise, amplitude-modulated tone, and frequency-modulated tone. Noriaki Asemi (R. I. E. C./ G. S. I. S., Tohoku Univ., 2-1-2 Katahira, Aoba-Ku, Sendai 980-8577, Japan), Yoichi Sugita (Natl. Inst. of Bioscience and Human-Technol., Japan), and Yôiti Suzuki (Tohoku Univ., Sendai, Japan)

To examine basic features of sounds used in the perception of sound environment, auditory search tasks seem promising to us. Thus the response time to detect a target sound among distracting sound(s) was measured by three experiments. In the first experiment, pure tones and narrowband noises (1/12 oct) were used. Either of them was used as a target and the other was used as distractor(s). The number of the distractor was varied from 1 to 6 . Each sound was presented from one of seven loudspeakers located on the horizontal plane. In the second and third experiments, pure tones and amplitude-modulated tones, pure tones and frequency-modulated tones, respectively, were used as either a target or distractor(s). Results show that the response time to detect a narrow-band noise, an amplitude-modulated tone, or a frequency-modulated tone among distracting pure tones was hardly affected by the number of the distractors. However, the time required to detect a pure tone increased with the number of distractors. These results indicated that our auditory system utilizes temporal changes in amplitude and frequency of sound as a basic feature for the detection of a sound in a sound environment. Precise experimental results as well as further consideration will be given in the presentation.

3aPP8. Perception of a secondary auditory image with three sound sources. Bernard T. G. Tan, Sing Hai Tang, and Gongqiang Yu (Phys. Dept. of NUS, Low Kent Ridge Rd., Singapore 117543, osa-head@nus.edu.sg)

The precedence effect with three sound sources, using loudspeakers placed in a horizontal plane at equal distances facing the subject, was investigated. In this three-source sound system, one of which is undelayed and the other two delayed, a listener facing these sources perceives an unexpected secondary auditory image in addition to the expected primary auditory image. The two delayed sources have time delays relative to the undelayed source within the range in which the precedence effect operates. The secondary auditory image is believed to be due to summing localization taking place between the two sound sources, which have been delayed, when the relative time delay between them is within the range for summing localization to occur.

3aPP9. The use of off-frequency information in a high-frequency binaural discrimination task. Steven van de Par (Philips Res. Labs. Eindhoven, Prof. Holstlaan 4, NL-5656 AA Eindhoven, The Netherlands and IPO-Ctr. for User-System Interaction, Eindhoven, The Netherlands), Constantine Trahiotis, and Leslie R. Bernstein (Univ. of Connecticut Health Ctr., Farmington, CT 06030)

The ability of listeners to discriminate between $N_{0} S_{\pi}$ and $N_{0} S_{0}$ stimuli was measured as a function of signal-to-noise ratio. Three types of Gaussian-noise maskers were employed: (1) broadband (300 to $6000 \mathrm{~Hz})$ (2) high-frequency (2900 to $6000 \mathrm{~Hz}$ ), or (3) low-frequency (300 to 3100 
$\mathrm{Hz}$ ). The signal was a $3-\mathrm{kHz}$ sinusoid. The overall patterning of the data supports the hypothesis that listeners can and do utilize "off-frequency" information to enhance binaural performance. The data also suggest that the off-frequency information within auditory filters centered above the frequency of the signal is especially salient.

3aPP10. Perceptual segregation of individual components from a complex tone via changes in interaural time delay (ITD). John F. Culling (School of Psych., Cardiff Univ., P.O. Box 901, Cardiff CF10 3YG, UK)

When all the components of a complex tone share a common interaural time delay (ITD), a single component can be made to perceptually segregate from the complex by shifting its ITD to a unique value. Segregation of different components in succession can evoke the perception of a melody [Kubovy et al., Science 186, 272-274]. A series of five experiments demonstrated that (1) the ITD transition is more responsible for the segregation effect than the uniqueness of the ITD of the segregated component, (2) independent change in ITD of one component relative to coherent change among the other components is not sufficient to cause segregation, (3) cyclic modulation of ITD causes segregation which is independent of modulation rate, (4) temporal gaps in the stimulus of the order of $100 \mathrm{~ms}$, and centered on the ITD transitions, can prevent segregation. These findings are consistent with an explanation based on sensitivity to within-channel reductions in interaural correlation within a sliding temporal window of around 100-ms duration; when different ITDs are temporally juxtaposed within a time frame of around $100 \mathrm{~ms}$, these ITDs are encompassed by the window's span and the wave forms within it do not correlate perfectly at either delay.

3aPP11. Perception of acoustic occlusion using body-scaled judgments. Michael S. Gordon and Lawrence D. Rosenblum (Dept. of Psych., Univ. of California, Riverside, Riverside, CA 92521, rosenblu@citrus.ucr.edu)

Occlusion has been found to compel visual perception of object position and scene analysis [G. A. Kaplan, Percept. Psychophys. 6, 193-198 (1969); W. H. Warren and S. Wang, J. Exp. Psychol. 13, 371-383 (1987)]. Auditory occlusion may also provide salient information about the locations of both sound sources and surfaces in an acoustic scene. In the current experiment, blindfolded participants were asked to listen to sound occluded by an aperture and judge whether unobstructed passage was afforded for various aperture sizes (e.g., W. H. Warren and S. Wang, 1987). Judgments were found to be highly accurate, suggesting the salience of occlusion information for auditory perception. Information from the relative loudness between aperture sizes may have contributed to this ability.

3aPP12. Head-slaved tracking of an acoustic target by human listeners. Robert S. Bolia and W. Todd Nelson (Air Force Res. Lab., 2255 H. St., Wright-Patterson AFB, OH 45433-7022)

Numerous researchers have investigated the extent to which humans are able to track a visual target simply by following its motion with corresponding motions of the head. The results of these investigations have many practical applications, including vehicle guidance and the tracking and designation of moving targets. In spite of the amount of work done in this area, few, if any, studies have addressed the question of how well humans are able to track an acoustic target. This is surprising, given that, in addition to its potential advantages for multi-sensory tracking displays, head-slaved tracking of acoustic targets might prove utile as a means of navigation for the visually impaired. In the present study, listeners performed a head-slaved tracking task of an acoustic target the motion of which was constrained to the horizontal plane, under factorial combinations of field-of-regard ( $\pm 30,45$, or 90 degrees), spatial resolution of the audio display (3, 7, or 10 degrees), and the presence or absence of visual feedback (a color change in the visual display). Results will be discussed in terms of potential applications, and compared with performance on visual tracking tasks. [Work supported by the Air Force Office of Scientific Research.]

3aPP13. Velocity DLs for a simulated sound source: Effect of intensity. Sarah Hassett and Lawrence Feth (Dept. of Speech and Hearing Sci., 110 Pressey Hall, Ohio State Univ., Columbus, OH 43210)

The Doppler effect refers to the frequency change observed for a moving sound source. The listener will observe a higher than emitted frequency as the moving source approaches, and a lower frequency as it recedes. Previous work [J. Acous. Soc. Am. 106, 2209 (1999)] determined the difference limen (DL) for the velocity of a computer-simulated moving sound source. The simulated source moved along a straight path that passed within $5 \mathrm{~m}$ of the listener. Subjects were tested at 500,1000, and $4000 \mathrm{~Hz}$ for reference velocities of 1, 2, 4, and $8 \mathrm{~m} / \mathrm{s}$. Signal duration was $500 \mathrm{~ms}$. A 2Q,2AFC adaptive procedure determined the listener's velocity DL. The results indicated that DLs for both velocity and inferred frequency increase as reference velocity decreased, indicating that Weber's law does not hold for either parameter. The influence of source intensity variations for the 5-m path were minimal, thus the current experiment repeated the conditions of the prior experiment except that the path passed $0.5 \mathrm{~m}$ from the location of the observer. The effects of simultaneous frequency and intensity changes were investigated and compared with model predictions. [Work supported by a grant from the Ohio State University College of Social and Behavioral Sciences.]

3aPP14. Influence of visual cues on the perception of surround sound. Andrzej Czyzewski, Artur Kornacki, Bozena Kostek, Piotr Odya, and Slawomir Zielinski (Sound Eng. Dept., Tech. Univ. of Gdansk, 80-952 Gdansk, Poland, kid@ sound.eti.pg.gda.pl)

Contemporary digital video, film, or multimedia presentations are often accompanied by the surround sound. Techniques and standards involved in digital video processing are much more developed than concepts underlying creating recording and mixing of the multichannel sound. The main challenge in the sound processing in the multichannel system is to create an appropriate basis for connecting multimodal context of visual and sound domains. Therefore one of the purposes of experiments is to study in which way and how the surround sound interferes or is associated with the visual context. This kind of study was hitherto carried out when two-channel sound technique was associated with a stereo TV. However, there are not many studies done yet that associate surround sound and digital video presented at the TV screen. The main issue in such experiments is the analysis of the influence of visual cues on perception of the surround sound. This problem will be addressed in the paper. 


\title{
Session 3aSA
}

\section{Structural Acoustics and Vibration and Noise: Combining Active and Passive Control of Vibration and Noise II}

\author{
Alain C. Berry, Cochair \\ Department of Mechanical Engineering, University of Sherbrooke, Sherbrooke, Quebec J1K 2R1, Canada \\ Thomas J. Royston, Cochair \\ Department of Mechanical Engineering, University of Illinois, 842 West Taylor, Chicago, Illinois 60607-7022
}

\section{Invited Papers}

8:30

3aSA1. Active stabilization of liquid capillary bridges using optically sensed modal amplitudes. David B. Thiessen, Mark J. Marr-Lyon, and Philip L. Marston (Dept. of Phys., Washington State Univ., Pullman, WA 99164-2814)

Cylindrical capillary bridges consisting of liquid between two circular supports naturally become unstable and break when the length of the bridge exceeds its circumference for the situation where the weight or buoyancy of the bridge can be neglected. This is the Rayleigh-Plateau (RP) slenderness limit which is relevant to the management of liquids and to the formation of liquid drops. We have demonstrated methods of suppressing this instability based on the optical sensing of the instantaneous modal amplitude and the rapid adjustment of the axial distribution of applied radial stress. This applied stress may be the result of ultrasonic radiation pressure [M. J. Marr-Lyon et al., J. Fluid Mech. 351, 345-357 (1997)] or electrostatic stresses from an array of electrodes [M. J. Marr-Lyon et al., Phys. Fluids (accepted)]. We have stabilized bridges as much as $42 \%$ beyond the RP limit by phasing the stress so that the effective modal spring constant becomes positive; however, the modal damping is then decreased. The closed-loop response includes terms associated with boundary-layer damping and the sensor's response time. The analysis indicates that damping of short bridges may be enhanced by reversing the feedback phase. [Work supported by NASA.]

8:50

3aSA2. Active structural intensity control using strain sensing. Pascal Audrain (G.T.V., I.A.M., Université du Maine, 72085 Le Mans Cedex 9, France, audrain@laum.univ-lemans.fr), Patrice Masson, and Alain Berry (Université de Sherbrooke, Sherbrooke, PQ J1K 2R1, Canada, Patrice.Masson@gme.usherb.ca)

An investigation of structural intensity control using strain sensing is presented in this paper. As opposed to previous work, the approach taken here does not assume waves propagating predominantly in one direction. Moreover, the instantaneous intensity is completely taken into account in the control algorithm, i.e., all the terms are considered in the real-time control process and, in particular, the evanescent waves are considered in this approach. A finite-difference approach using discrete PVDF strain sensors is used as the sensing scheme. A feedforward filtered-X LMS algorithm is adapted to this energy-based control problem, involving a nonpositive definite quadratic form in general. In this respect, the approach is limited to cases where the geometry is such that the intensity component will have the same sign for the control source and the primary disturbance. Experimental validation of the approach is conducted on a free-free beam covered with viscoelastic material. A comparison is made between classical acceleration control and structural intensity control and the performance of both approaches is presented. These results tend to indicate that using intensity control allows the error sensors to be placed closer to the control source and the primary disturbance, while preserving a good control performance.

\section{Contributed Papers}

9:10

3aSA3. Progress toward a "smart acoustic blanket." Joseph A. Bucaro, Brian H. Houston, Thomas Howarth, Robert Corsaro, James Tressler, and Nicholas Lagakos (Naval Res. Lab., Washington, DC 20375-5350)

This paper presents recent progress we have made toward the goal of developing what we call "smart acoustic blankets." Such technology would have application in many acoustic areas including aeroacoustics, architectural acoustics, and traffic sound barriers in addition to our principal focus which is the reduction of launch noise sound transmission through satellite payload fairings. These smart blankets would be configured with sound actuation layers, acoustic pressure and velocity sensors, and electronic controller modules. The status of the development of each of these components as well as the overall concept will be discussed. [Work supported by ONR.]
9:25

3aSA4. Hybrid tool for quick characterization of multi-layered panels. Ibrahima Sow, Olivier Beslin, and Jean Nicolas (GAUS, Dept. Genie Meca., Univ. of Sherbrooke, Sherbrooke, PQ J1K 2R1, Canada, isow@vulcain.gme.usherb.ca)

With the advancement of science and technology, materials with high damping capabilities and high modulus of elasticity are increasingly popular as they reduce vibration and noise. However, most of the time, there is a lack of tools that allow quick and easy characterization of the effective materials properties when used in 2-D structures. In this paper, a method is proposed for the characterization and analysis of viscoelastic composite material consisting of a three-ply sandwich panel. The method is based on a quick PC-based numerical code (involving hierarchical finite element method coupled with Beraneks formulation of sandwich panels) and a dedicated simple experimental setup. This approach ensures (i) proper extraction of the equivalent properties such as the modulus of elasticity, 
the loss factor according to temperature, or frequency of the entire sandwich composite, (ii) characterization of any core viscoelastic resin, and (iii) evaluation of vibroacoustics indicators. The method is validated using commercially available 3M ISD112 and good agreement is found between experimental and numerical results. The method is then used to carry out a parametric study for different panel configurations according to temperature and frequency in order to characterize a viscoelastic resin.

\section{9:40}

3aSA5. Electro-elastic laminate theory for discrete piezoelectric patches on laminated plates. Senthil Gopinathan, Vasundara Varadan, and Vijay Varadan (Penn State Univ., 149 Hammond Bldg., University Park, PA 16802)

Classical laminated plate theory (CLT) has been applied successfully in the past to laminates with discrete piezoelectric patches bonded to the surface or embedded within the layers (1-7). The basic assumptions made in the earlier models were that the strains inside the patches are assumed to be constant and hence the presence of the sensor and actuator patches were neglected while modeling the dynamic properties of the laminate. The validity of these assumptions, the effect of the size of the patches and these assumptions on the solutions obtained, has not been studied. In this paper, the CLT is applied to a laminate with surface-bonded piezoelectric patches without the above-mentioned assumptions. A detailed modeling of the patches is developed by expressing the electric potential inside the patch as a quadratic function of thickness coordinate. The equations of motion are derived for a generally orthotropic laminate and solution method for these equations. Analytical solutions are obtained for a plate bonded with one and five collocated piezoelectric actuator/sensor patches. The effect of the passive and active stiffness of the surface bonded actuator and sensor patches on the dynamic characteristics of host plate structure is studied.

\section{9:55}

3aSA6. Structural intensity in a line-excited infinite elastic plate in the high-frequency range. Sabih I. Hayek and Jungyun Won (Active Vib. Lab., Penn State Univ., 227 Hammond Bldg., University Park, PA 16802 sihesm@engr.psu.edu)

An infinite elastic plate is excited by a mechanical line force, which generates an active structural intensity field in the plate. In an earlier paper [Hayek et al., J. Acoust. Soc. Am. 105, 1299 (1999)], the structural intensity was evaluated analytically for a plate using the Bernoulli-Euler theory, which is valid in the low-frequency range. The paper then employed the same theory for the active control of total structural intensity by use of colocated point forces and moments. In this paper, the evaluation of the structural intensity field due to line forces and moments is modeled by use of the more exact Mindlin plate theory, which is valid in a much higher frequency range. The main objective is to achieve active control of the total structural intensity in infinite elastic plates using the Mindlin plate theory through a colocated line force and a line moment actuators at an arbitrary location on the plate.

\section{0:10-10:30 Break}

\section{0:30}

3aSA7. Impact of piezoceramic transducer hysteresis in the hybrid structural vibration control problem. Soon-Hong Lee and Thomas J. Royston (Dept. of Mech. Eng., MC251, Univ. of Illinois at Chicago, 842 W. Taylor St., Chicago, IL 60607)

Hybrid vibration control via passive electrical shunting and active actuation of a piezoceramic wafer bonded onto the surface of a simply supported beam is investigated theoretically and experimentally with emphasis on modeling and understanding the impact of the piezoceramic hysteresis. A nonlinear rate-independent hysteresis model is experimentally identified for the PZT wafer by itself and then integrated into the coupled dynamic equations of the overall system consisting of the beam and electrically shunted PZT wafer. Experimental studies of the system validate the theoretical model. This model is then used to investigate the impact of PZT hysteresis on its vibration control performance in passive, active, and hybrid scenarios. [Work supported by grants from ONR and NSF.]

\section{0:45}

3aSA8. A new active structure acoustic control system. Dingguo Zou and Malcolm J. Crocker (Dept. of Mech. Eng., Auburn Univ., Auburn, AL 36849, zouding@eng.auburn.edu)

The acoustic power radiated from any vibrating structure can be written as a quadratic form of the structural modal velocities. The quadratic matrix is real, symmetric, and positive definite. All of the terms with even-odd and odd-even indices are zero. The congruent transformation instead of orthonormal transformation was used to diagonalize the matrix. This method takes advantage of the good properties of the quadratic matrix more efficiently than the orthonormal transformation does. Also, the method greatly simplifies the design of the PVDF sensors, which filter the combination of appropriately weighted vibration modes, providing a specific performance index in control strategy. Based on this method, an active control system was implemented. To minimize the radiated power from a vibrating structure, a procedure to optimize the distribution of the actuators was applied to the control system.

\section{1:00}

3aSA9. Sensing radiated acoustic power using smart sensors. Dingguo Zou and Malcolm J. Crocker (Dept. of Mech. Eng., Auburn Univ., Auburn, AL 36849, zouding@eng.auburn.edu)

This paper represents the radiated acoustic power from a vibrating structure as a quadratic form of the structural modal velocities. The weighting matrix is diagonalized by using the congruent transformation. A group of one-dimensional shaped smart sensors which can sense the acoustic power from the structure by filtering the combination of weighted vibration modes are designed. First, it was found that if the shaped function is chosen carefully, the measuring from a two-dimensional distributed parameter sensor using PVDF film is independent of the position. Then, the plate is vertically divided by some narrow strips. The narrow strips are assumed to be approximate one-dimensional plate. The one-dimensional shaped sensor equations can be used to measure a particular modal coordinate. The acoustic power can be obtained by weighting the measurements from multiple one-dimensional smart sensors. Finally, the approximate results are adjusted further by using the theoretical formulation.

\section{1:15}

3aSA10. Active vibration control of a structure using virtual sensors. Vivian F. Dias and Scott D. Sommerfeldt (Dept. of Phys., Brigham Young Univ., Provo, UT 84602, vdias@physics.byu.edu)

Presently, a large safety factor is used in determining routine maintenance schedules for critical machinery/parts, such as replacement of skins for aircraft wings, because any failure during operation could prove catastrophic. Assuming that the location of the structural damage can be identified at its inception, one could apply active vibration control on the structure as a means of reducing stresses in the damage zone, thus ensuring that the damage does not grow rapidly with time. However, the probability of having an error sensor at the damage location is small, which indicates that a different approach would be required for implementing the active control. Work will be reported aimed at developing an active vibration control system that allows the control to minimize the stress or vibration at any arbitrary point/zone on the vibrating structure, even where there is no error sensor present. This is accomplished by using what are referred to as "virtual sensors." These virtual sensors use information from physical sensors at other locations on the structure to estimate the vibration response at the virtual sensor location. This approach offers the possibility of being able to control the structural vibration at any desired, arbitrary location on the structure. 
3aSA11. Active/passive design for vibration control. John A. Rule and Robert L. Clark (Dept. of Mech. Eng. and Mater. Sci., Duke Univ., Durham, NC 27708, jarule@duke.edu)

A method has been developed for the control of panel vibration using a hybrid active/passive spatial optimization technique. The method relies on the rapid estimation of Hankel singular values (HSV's) to predict coupling of sensor/actuator pairs to particular vibration modes of interest. The two-step process first requires the selection of the best sensor/actuator pair for active control, using a design metric which is typically used to target the control of low-frequency, long-wavelength modes, while simultaneously minimizing higher frequency, or out-of-bandwidth, system response. With the active control system in place, potentially destabilizing out-of-bandwidth modes are identified for passive control. A similar design metric is then applied which emphasizes coupling of passive damping material to the high-frequency, short-wavelength structural modes. Numerical simulations were performed to demonstrate this concept. It was then implemented on an $18-\times 16$-in steel plate. Plate response was measured first for the uncontrolled plate, then the plate with just the active control system in place, and finally the complete hybrid active/passive system. Performance of each system was analyzed, with benefits and tradeoffs considered.
3aSA12. Active vibration isolation using variable structure control strategy. Jiaqiang Pan and Shuwen Pan (Dept. of Instrumentation Sci. and Eng., Zhejiang Univ., Hangzhou 310027, PROC)

In this paper, the authors present a new type of control design methodology used for active vibration isolation: Variable Structure Control (VSC) with sliding mode. VSC is a special control strategy that is capable of making a control system very robust with respect to system parameter variations and external disturbances, and providing an easy way to design the control law for a plant, linear or nonlinear. It has developed into a general control design method and been applied to a wide variety of engineering systems since the 1990s. In this study, a vibration isolation system with both active and passive isolators is designed based on Ackermann's method. Using this method, designed control law can make system states move in a time-variant sliding mode surface in state space from initial moment. This ensures the stability and robustness of the closed-loop system. Designed feedback control law can be expressed explicitly by state variables and parameters of the nominal system without any uncertainty and is easily applied in practice. Simulation results of an active suspension of vehicles show that the control system using VSC strategy possesses good performance.

\title{
Session 3aSC
}

\section{Speech Communication: Phonetics, Prosody and the Lexicon (Poster Session)}

\author{
Michael D. Hall, Chair \\ Psychology Department, University of Nevada, 4505 Maryland Parkway, Box 455030, Las Vegas, Nevada 89154-5030
}

\section{Contributed Papers}

\begin{abstract}
All posters will be on display from 8:30 a.m. to 11:30 a.m. To allow contributors an opportunity to see other posters, contributors of odd-numbered papers will be at their posters from 8:30 a.m. to 10:00 a.m. and contributors of even-numbered papers will be at their posters from 10:00 a.m. to 11:30 a.m.
\end{abstract}

3aSC1. Speech perception by normal-hearing listeners under modulated noise masker. Bom Jun Kwon and Christopher W. Turner (Dept. of Speech Pathol. and Audiol., Univ. of Iowa, Iowa City, IA 52242, bomjun-kwon@uiowa.edu)

Previous psychoacoustic investigations on modulation detection interference (MDI) show that sensitivity to detect modulation in a target is reduced when the masker is modulated. The amount of reduction is primarily dependent upon modulation frequencies, but not as much upon carrier frequencies, supporting the existence of a modulation filterbank in the auditory system. In the current study, consonant identification by normal-hearing subjects was tested with noise maskers, either steady-state or modulated. Modulation interference (MI) was assessed by comparing recognition under the two masker types. In experiment I, speech with limited envelope modulations was tested with different rates of masker modulation. In experiment II, narrow-band speech and masker were tested under various carrier frequency conditions. Higher MI was observed when the rate of masker modulation lies within the range of speech modulations. Carrier frequency separation did not make a strong effect on MI except when the separation is zero. In many cases MI was small or negative, implying that a masking release also occurred. In conclusion, both modulation masking and masking release should be taken into consideration when addressing speech perception under modulated maskers. Psychoacoustic MDI results, therefore, only partially predict the effects of modulation interference on speech recognition. [Work supported by NIDCD.]
3aSC2. An EPG and acoustic study of fricative production

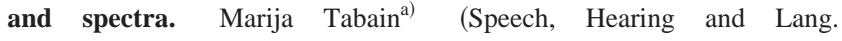
Res. Ctr., Macquarie Univ., Sydney, 2109, Australia, marija@srsuna.shlrc.mq.edu.au)

In order to test the correspondence between variability in fricative production and variability in the fricative spectrum, the spectral center of gravity (COG) is compared with the EPG center of gravity measured along the horizontal dimension. The CV coarticulatory effects in particular are examined. Data are taken from CV tokens produced by four female speakers of Australian English. Tokens consisted of the coronal fricatives /T s S D z Z/ in seven monophthong vowel contexts. Rounded vowels are excluded so as to minimize spectral changes due to lip-rounding. The consistency between the articulatory and the acoustic data is particularly striking for the palato-alveolar fricatives, although results are also highly consistent for the other two fricative places of articulation examined. However, it is not always clear that the variability in fricative production is conditioned by the vowel context. Overall, the sibilant fricatives seem particularly resistant to coarticulation and to variability in production, while the nonsibilant dental shows a good deal of variability, including some conditioning by the vowel context. These results have implications for the hyper- and hypo-theory of speech production. It is suggested that sibilant fricatives do not lend themselves to the articulatory imprecision, 
which characterizes other perceptually salient, and typologically common, speech sounds. ${ }^{\text {a) }}$ Currently at Perceptual Science Laboratory, University of California at Santa Cruz.

3aSC3. Comparative perception of voicing contrasts. Joan M. Sinnott and Laura A. McArdle (Psych. Dept., Univ. of South Alabama, Mobile, AL 36699,jsinnott@jaguar1.usouthal.edu)

English, Spanish, and monkey listeners were compared in their perception of a synthetic labial VOT continuum $(-60$ to $+70 \mathrm{~ms})$. A goright/go-left ID procedure was used, with food reinforcement for monkeys. In exp. 1, subjects first learned to identify the endpoints of the continuum as "BA" or "PA," and then generalization responses were obtained for intermediate stimuli. The mean boundary for English listeners was at $+25 \mathrm{~ms}$, while boundaries for Spanish listeners ranged from -15 to $+25 \mathrm{~ms}$ (mean=7.6 ms). The monkey boundary was near $0 \mathrm{~ms}$ and appeared to simply bisect the continuum, suggesting that the English boundary at $+25 \mathrm{~ms}$ is not a salient feature of the "general auditory system" in the context of prevoiced stimuli. In exp. 2, subjects were trained to differentiate VOT contrasts that crossed either the "English" or the "Spanish" boundary. While subjects learned to reliably differentiate both contrasts, reaction times were fastest for the English contrast. These results indicate that although attentional differences between subject groups may influence their generalization responses along a VOT continuum (exp. 1), these groups have similar sensory capacities when explicitly trained to differentiate specific stimuli along a VOT continuum (exp. 2). [Work supported by NIDCD.]

3aSC4. Coproduction in VCV disyllables produced by children and adults. Carole E. Gelfer (Dept. of Comm. Disord. William Paterson Univ., Wayne, NJ 07470, gelferc@wpunj.edu) and Fredericka Bell-Berti (St. John's Univ., Jamaica, NY 11439)

Previous studies of speech production of young children have suggested differences from adult productions. For example, developmental differences have been noted for VOT and the extent of coarticulation between vowels and fricatives. This study explores developmental trends for consonant-vowel interactions in VCVs disyllables as evidenced by spectral differences in V1 as a function of C and V2 identity, where V1 is schwa, C is one of the following: /p, t, k, b, d, g, s, sp, st, sk/, and V2 is /i/ or /u/. The children in this study are between the ages of 4 and 6 . Measures of $F_{2}$ at both the offset and midpoint of schwa will be used to determine (1) the extent to which interactions show developmental trends, and (2) whether the extent of these interactions for either or both groups of speakers is influenced by consonant manner of articulation, place of articulation, and/or duration.

3aSC5. Anticipatory coarticulation in the speech of adults and children: A perceptual study. Sneha V. Bharadwaj and William F. Katz (Univ. of Texas at Dallas, Callier Ctr. for Commun. Disord., 1966 Inwood Rd., Dallas, TX 75235)

Two competing theories attempt to explain anticipatory coarticulation in the speech of adults and children: One suggests more extensive coarticulation in children's speech than in that of adults, while the other suggests more extensive coarticulation in adult speech [see S. Nittrouer and D. Whalen, J. Acoust. Soc. Am. 86, 1266-1276 (1989) for discussion]. To address this issue, a perceptual experiment was conducted in which adult listeners identified vowel information from gated CV syllables. The syllables /si/, /su/, / $\mathrm{i} /$, and //u/ produced by children (5 and 7 years old) and adults were gated into four segments of varying lengths ( $\frac{1}{2}$ fricative, $\frac{3}{4}$ fricative, full fricative, and fricative $+\frac{1}{2}$ vowel). The stimuli were presented to ten adult listeners in randomized order (experiment 1) and to another ten listeners, blocked by individual talkers (experiment 2). Syllable identification for key fricative gates was $6 \%$ better in blocked compared to random presentation. Both experiments revealed a trend of listen- ers detecting more extensive coarticulation in children's productions than in those of adults; however, this pattern resulted from heightened responses to a single 7-year-old talker. Results suggest children coarticulate with greater variability than adults, but not necessarily with a greater temporal extent.

3aSC6. Threshold comparisons for intensity-based duplex perception for speech. Michael D. Hall (Psych. Dept., Univ. of Nevada Las Vegas, 4505 Maryland Pkwy., Box 455030, Las Vegas, NV 89154-5030, hallm@nevada.edu)

Duplex perception (DP) occurs when a stimulus component simultaneously contributes to two percepts (Rand, 1974). A proposed DP variant involves substituting a tone glide for an F3-transition in a /da/ or /ga/ syllable. At full intensity the glide is perceived as a nonspeech chirp that simultaneously distinguishes the consonant. It also has been claimed that the glide contributes to speech (consonant) perception over a range of intensities that are insufficient for nonspeech (chirp) detection, raising the possibility that a phonetic module takes precedence over nonspeech perception (Whalen and Liberman, 1987). Others have failed to find a range of precedence when chirp detection thresholds were reevaluated using a 2AFC task (Bailey and Herrmann, 1993). Which conclusion is valid? The current investigation addressed this question by comparing individual thresholds for consonant perception and chirp detection using a variety of methods. Chirp detection thresholds were evaluated using the method of constant stimuli, 2AFC, and various reminder tasks (using a fixed standard). Thresholds for consonant perception were evaluated using identification and discrimination (same-different) tasks. Results analyzed according to both traditional threshold and detection theories support the claims for a range of precedence and suggest that discrepant chirp detection thresholds reflect a reliance on consonant perception.

3aSC7. Vowel reproduction exhibits an intrinsic dynamic organization. Betty Tuller and Gautam K. Vallabha (Ctr. for Complex Systems \& Brain Sci., Florida Atlantic Univ., 777 Glades Rd., Boca Raton, FL 33431, vallabha@walt.ccs.fau.edu)

Speech perception and production are generally thought to be tuned by the speaker's linguistic environment. If so, iterative reproduction of a vowel by a single speaker may amplify subtle biases introduced by the tuning. Four male monolingual speakers of American English were asked to reproduce 100 synthetic vowel-like stimuli, uniformly distributed in a two-formant acoustic space ( $F 1, F 2$ varying, $F 0=120 \mathrm{~Hz}, F 3=2500 \mathrm{~Hz}$, duration $=200 \mathrm{~ms}$ ). Fifteen of the subject's own productions were chosen for serial reproduction, as follows: Each original production was presented to the subject through headphones, and the subject immediately mimicked it. After a 0.5-s delay, the "mimic" was played back to the subject as the target for his next production. This second mimic was the target for the following production, and so on, for ten iterations. Subjects made systematic errors in reproducing their own vowel sounds, and sequences of serially reproduced vowels behaved differently in different parts of the vowel space. These results suggest that some vowel regions are preferred over other regions, indicating that the cognitive processes for perceiving and producing vowels have complex intrinsic dynamics. [Work supported by NIMH.]

3aSC8. Consonants and vowels behave differently in silent center syllables. A. Min Kang (Haskins Labs., 270 Crown St., New Haven, CT 06511 and Dept. of Linguist., Yale Univ., New Haven, CT 06520, min.kang@yale.edu) and D. H. Whalen (Haskins Labs., New Haven, CT 06511)

Compared to consonants, steady-state vowels show no right ear advantage, activate different brain regions, and only trigger categorical perception when extremely short. Auditory explanations for this distinction assume different perceptual mechanisms for brief, dynamic consonants and 
long, slow-changing vowels. Gestural views suggest steady-state vowels are perceived as only partially speechlike. The present study compares consonant and vowel identification and discrimination when based on the same brief, dynamic information in the form of silent center (SC) syllables. The CVCs simultaneously varying in equal-sized $F 2$ steps along both a $/ \mathrm{b}-\mathrm{d} /$ and an $/ \epsilon-\wedge /$ continuum were synthesized (full syllables). The SC syllables were produced by replacing the middle $60 \%$ with silence. Relative to full syllables, overall SC consonant discrimination improved from chance to $70 \%$; vowel discrimination dropped from $85 \%$ to $70 \%$. The SC vowel identification was affected by consonantal context but the reverse effect was weaker. Improved SC consonant discrimination may result from decreased masking or greater prominence of the consonant gesture. Decreased SC vowel discrimination could result from reduced acoustic information or weaker prominence of the inferred vowel gesture. While these results do not distinguish auditory and gestural theories, they do place the consonant/vowel distinction in a new perspective. [Work supported by NIH Grant No. HD-01994.]

3aSC9. The role of sentential prosody in learning voices. Lynne C. Nygaard and Jennifer S. Queen (Dept. of Psych., Emory Univ., Atlanta, GA 30322, lnygaar@emory.edu)

The present experiment was designed to investigate the contribution of sentential prosody to the perceptual learning of talker's voice. Listeners were trained over a three-day period to learn talker's voices from three types of utterances: (1) sentence-length utterances produced with natural sentential prosody, (2) utterances in which individual words were produced in list format and digitally assembled into complete sentences, and (3) words produced in list format and scrambled to remove semantic and syntactic coherence. Equal numbers of male and female listeners learned a set of ten talker's voices (five male and five female) and learning was evaluated at each training session. On the fourth day of testing, listeners were given a generalization test that consisted of novel sentence-length utterances produced with natural sentential prosody. Results indicated that learning curves were comparable across conditions, but the ability to generalize to new utterances depended on the utterance type used during training. Individual differences among both listeners and talkers were also observed. Male listeners identified male voices significantly better than female voices. Female listeners identified male and female voices equally well. These results suggest that the perception of talker identity is dependent both on listener- and task-specific characteristics.

3aSC10. Perceptual adjustment to foreign-accented English. Constance M. Clarke (Dept. of Psych., Univ. of Arizona, Tucson, AZ 85721)

A central goal of speech research is to understand how the speech perception system handles variability. Previous work on variability due to differences in speakers' voices has demonstrated that experience with a voice facilitates word intelligibility [L. C. Nygaard and D. B. Pisoni, Percept. Psychophys. 60, 355-376 (1998)]. The present study investigated whether a more abstract feature of voices, such as foreign accent, can also be perceptually learned and utilized in perception. The following question was tested: Does experience with an accent generalize to the perception of a new voice with the same accent? Two groups were trained with English sentences produced by either Spanish- or Chinese-accented voices. Both groups were then given a word intelligibility test in which sentences were presented in noise. Test sentences included both familiar and new Spanish- and Chinese-accented voices. Training improved intelligibility of familiar voices but did not affect perception of new voices with the trained accents. Results indicate that experience with an accent does not improve perception of a new voice with the same accent. Perceptual learning seems to be restricted to individual voices. Results are discussed with respect to current theories of speech perception. [Work supported by NSF.]

3aSC11. Imitation of phrases in conversational speech. Jennifer S. Pardo (Psych. Dept., Yale Univ., P.O. Box 208205, New Haven, CT 06520, jennifer.pardo@yale.edu) and Carol A. Fowler (Haskins Labs., New Haven, CT 06511)

Linguistically significant gestures of the vocal tract play an important role in speech perception and production. Imitation of speech across conversational partners is a natural consequence of the close connection between perception and production. Following research that finds imitation in single-word shadowing, this study paired unacquainted same-sex talkers in a conversational task designed to induce repetition of key phrases across partners. Immediately repeated items were assessed for imitative fidelity by asking a separate set of listeners to choose the more similar item in an AXB task that compared the sample item from one talker (X) with a read and a repeated item from the other talker (A/B). In general, listeners chose the repeated over the read item as more similar to X. Additionally, read items that were sampled immediately after the conversation hindered a listener's ability to choose the repeated item more so than items read long before the conversation. Other effects related to the timing of items in the conversation and the role of the participants in the conversation were also observed.

3aSC12. Age-related differences in regularity of speech rate. Richard J. Morris (Dept. of Commun. Disord., Florida State Univ., Tallahassee, FL 32306-1200, rmorris@mailer.fsu.edu)

Older adults speak more slowly and exhibit greater speaking rate variability than do young adults [i.e., Liss et al., J. Geron. 45, P35-45 (1990); Ramig, J. Commun. Disord. 16, 217-226 (1983)]. These findings have been reported for rates of speech, articulation, and reading as well as for individual consonants and vowels. However, one recent work found that their group of older adults exhibited greater regularity in their repetition of syllables [Watson and Fozo, ASLHA Conv. (November, 1999)]. These authors hypothesized that the older speakers may have less flexibility in the timing of their speech. The purpose of the present study was to test the hypothesis of reduced timing flexibility in the speech of older people. Ten older men and ten younger men served as subjects for this study. All subjects were in good general health and had normal hearing for their age. The subjects were directed to repeat a series of single syllable words, pat', bat', sack' and shack', at slow, normal and fast rates. The speech was recorded onto a DAT recorder and analyzed using the CSpeech software. Results are discussed in terms of age differences in speech timing across the three speaking conditions.

3aSC13. The role of prosody in scope relations. Mary Baltazani (UCLA Linguist. Dept., Los Angeles, CA 90024, marybalt@ucla.edu)

It is well known that sentences with two quantifiers or operators can be ambiguous depending on their scope relation. For example, "She didn't kick him because he screamed" can mean "His screaming prevented her kicking him" (because > not) or "His screaming was not the reason for her kicking him"' (not $>$ because). This paper reports on a production and a comprehension experiment on the role of prosody in disambiguating scope relations in Greek sentences. In the production experiment, five participants read aloud seven sentences each, containing negation and another operator ("because" or a quantifier like "two"), embedded in disambiguating contexts. Prosodic analysis of the utterances showed that speakers produced different prosodic structures for the two interpretations: a big prosodic boundary after negation to deliver the quantifier $>$ negation interpretation, and no boundary for the reverse interpretation. In the comprehension study, 40 participants listened to the 35 sentences out of context and performed a forced-choice paraphrase selection task. The results 
show a significant correlation between prosodic cues and scope disambiguation in Greek. This suggests that both speakers and listeners unconsciously make use of these prosodic cues to interpret sentences.

3aSC14. Lexical access in a divided attention task: Evidence from a new verbal transformation methodology. Peter W. Lenz, Richard M. Warren, and James A. Bashford, Jr. (Dept. of Psych., Univ. of Wisconsin-Milwaukee, Milwaukee, WI 53201, bashford@uwm.edu)

A recorded repeating word undergoes illusory changes (Verbal Transformations, or VTs) to forms consisting of syllables and words occurring in the listener's lexicon. When the repeating word is delivered dichotically with a long interaural delay, the listener hears two distinct lateralized images, each changing independently [see Warren and Ackroff, Nature (London) 259, 475-477 (1976)]. The present study introduces a new methodology minimizing vigilance and memory demands posed by the traditional VT paradigm. Rather than continuous monitoring and reporting of changes occurring on each side, listeners simply reported the forms being heard when cued by a light flash. In keeping with earlier findings based upon continuous bilateral monitoring, the simultaneous organizations of the same word were found to change independently. Contrasting with earlier studies, however, the presence of a second image of the same word did not decrease the transition rate, presumably due to reduced task demands. Interestingly, the transition rate decreased dramatically when two different repeating words were presented. Implications of these findings regarding mechanisms employed in processing speech will be discussed. [Work supported by NIH.]

3aSC15. Phoneme restoration in infants. Rochelle $S$. Newman (Dept. of Psych., E11 Seashore Hall, Univ. of Iowa, Iowa City, IA 52242, rochelle-newman@uiowa.edu)

In a classic study, Warren (1971) replaced the medial /s/ in legislatures with a cough, and found that adult listeners heard the word as being complete. This auditory illusion demonstrates effects of both bottom-up and top-down information: it occurs only when there is a possible masking noise (it does not occur if the /s/ is replaced with silence), and it occurs at least in part because of the listener's knowledge that legislatures is a real word. Thus, it involves an interaction between multiple sources of information. The present study investigates whether infants will also show such an effect, and thus whether this interaction between levels of language processing is itself something which develops over time. Infants are shown videos of a cat and a dog in a preferential-looking paradigm, and hear the words kitty and doggie both in the clear, and with the medial stop consonant replaced by either noise or silence. Results examine whether infants will treat the words as whole (that is, match them to the appropriate referent) when the middle sound is replaced with noise as compared to when it is replaced with silence. [Work supported by NIH Grant R03 HD37822-01 to the University of Iowa.]

3aSC16. Phonetic metamorphosis of white noise: A stop or a fricative. Valeriy Shafiro (City Univ. of New York Grad. Ctr., Dept. of Speech and Hearing, 365 5th Ave., New York, NY 10016)

The role of spectro-temporal coherence, lexical status, and word position in the perception of speech in acoustic signals containing a mixture of speech and nonspeech sounds was investigated. Stimuli consisted of nine words in which either white noise was inserted only into the silent interval preceding the onset of vocalic transitions ambiguous between /p/ and /f/, or in which white noise was mixed with the entire speech signal. A control condition contained no noise. Ten adults were asked to decide whether they heard a word with a /p/ or /f/ as the phoneme in the initial, medial, or final word position. The subjects perceived /f/'s $85 \%$ of the time when noise was inserted into the silent interval, $47 \%$ when noise was presented over the entire word, and $1 \%$ in the control condition. These results indicate the importance of spectro-temporal coherence for perceiving speech in a mixture of sounds. The lexical status and position of the critical phoneme in the stimuli also appeared to affect subjects' responses, although to a smaller degree. Theoretically, the results support the auditory scene analysis view of perceiving speech in heterogeneous signals, while they cannot be adequately explained by the motor theory of speech perception.

3aSC17. A comparison of the vowel sequence illusion and the verbal transformation effect. Jeffrey M. Cooley, Richard M. Warren, and James A. Bashford, Jr. (Dept. of Psych., Univ. of WisconsinMilwaukee, Milwaukee, WI 53201, bashford@uwm.edu)

When repeated sequences of steady-state vowels have durations of their phonetic components below the 100-ms threshold for identification of order, the phonemes lose their identity. The sequences are heard as syllables occurring in the listener's lexicon (the Vowel-Sequence Illusion). When listening to repeating words, listeners also hear illusory syllables occurring in their lexicon (the Verbal-Transformation Illusion). Verbal transformations require the decay (satiation) of the initial veridical organization before the repetition-induced shifts in criteria permit nonveridical forms to be heard: The Vowel-Sequence Illusion does not require initial verbal satiation, and hence permits repetition-induced criteria shifts to tap directly into the lexicon-based syllabary. The intrinsic differences in the nature of the phonetic stimuli producing the two illusions result in characteristic distinctions between the organizations heard in the two illusions. Among these distinctions is the spectral splitting of the vowel sequences into two simultaneous organizations, one consisting of components above and the other below the 1500-Hz crossover frequency encountered in other contexts. This and other aspects of the two verbal illusions will be compared and contrasted, and demonstrations of both will be available. [Work supported by NIH.]

3aSC18. The relative time course of neighborhood and lexical effects. Rochelle S. Newman (Dept. of Psych., Univ. of Iowa, E11 Seashore Hall, Iowa City, IA 52242), James R. Sawusch, Paul A. Luce (Univ. at Buffalo, Buffalo, NY 14260), and Amanda Aubin (Univ. of Iowa, Iowa City, IA 52242)

We have previously demonstrated that neighborhood density can have effects similar to those of lexical status in phoneme identification tasks. In a typical task demonstrating a lexical effect, subjects might hear two series, one ranging from "bag" to "pag," while the other varies from "pal" to "bal." Subjects are more likely to classify ambiguous stimuli from each series as members of the category that makes a word (they would classify ambiguous items as " $b$ " in the bag-pag series but as " $p$ " in the pal-bal series). In a similar task where none of the four endpoints were words, subjects classified ambiguous items as members of the category that had a greater neighborhood density. The current study examines the relative time course of these effects. We created both lexical and neighborhood series using the identical initial contrast, and presented them to the same set of listeners. Neighborhood effects occurred primarily in the fastest responses, while lexical effects occurred in the medium and slower responses. That is, neighborhood effects appear earlier in processing, and dissipate quickly, whereas lexical effects take longer to build up. These results will be discussed in terms of their implications for models of word recognition.

3aSC19. Separable effects of neighborhood density and phonotactic probability on word recognition in speech. Nathan R. Large and Paul A. Luce (Dept. of Psych. and Ctr. for Cognit. Sci., Univ. at Buffalo, 245 Park Hall, Buffalo, NY 14260-4110, nathan@deuro.fss.buffalo.edu)

Previous research on the effects of probabilistic phonotactics on spoken word recognition has typically confounded phonotactics and similarity neighborhood density. In the present research, the inhibitory effects of density and facilitative effects of probabilistic phonotactics on spoken 
word processing were manipulated simultaneously. Four sets of monosyllabic words and nonwords were created by orthogonally combining two levels of neighborhood density and two levels of probabilistic phonotactics. Speed and accuracy were examined in three different experimental paradigms: single-word shadowing, A-X same-different matching, and speeded neighbor generation. Words and nonwords were either blocked by lexicality or intermixed. Blocking and mixing of stimuli were used to manipulate participants' focus on lexical and sublexical levels of processing. In part, our results demonstrate simultaneous effects (inhibitory and facilitative) of both density and phonotactics. The implications of our results for models of spoken word recognition and lexical access will be discussed.

THURSDAY MORNING, 1 JUNE 2000

AMERICAN ROOM, 8:00 TO 11:15 A.M.

\title{
Session 3aSP
}

\section{Signal Processing in Acoustics and Underwater Acoustics: Algorithms and Performance I}

\author{
Charles F. Gaumond, Chair \\ Naval Research Laboratory, 4555 Overlook Avenue S.W., Washington, D.C. 20375-5320
}

\section{Contributed Papers}

8:00

3aSP1. Assessing the relative detection performance of matched-field processing and conventional beamforming. Brian H. Maranda and David J. Thomson (Defence Res. Establishment Atlantic, P.O. Box 1012, Dartmouth, NS B2Y 3Z7, Canada)

The primary advantage of matched-field processing (MFP) over conventional plane-wave beamforming is the ability to localize an acoustic source in a single data snapshot; for example, MFP can help to decide whether or not the source is submerged. It is to be expected that MFP will also provide improved detection performance over the plane-wave beamformer when the acoustic pressure field at the array exhibits the complicated behavior that is typically observed in the oceanic waveguide. Accurately quantifying the performance gain is difficult, owing to analytical problems in determining the exact performance of a detector that thresholds many correlated search cells. However, on the basis of simple statistical arguments concerning the effective dimensionality of the search spaces, it is claimed that a rough comparison of detector performance is provided by the ratio of the quadratic forms that are used as the decision variables. This ratio is computed for several array and ocean scenarios, including both a vertical line array in shallow water and a horizontal line array in deep water. Two-dimensional plots (depth and range) are used to illustrate the detection gain that can be provided by matched-field processing.

8:15

3aSP2. Theoretical first-order bias and second-order variance for localization estimates from matched-field processing methods: Randomized signal. Aaron Thode, Eran Naftali, and Nicholas C. Makris (MIT, 77 Massachusetts Ave., Cambridge, MA 02139)

The bias and variance of range and depth estimates obtained from matched-field processing (MFP) methods are difficult to obtain analytically. Instead, the Cramer-Rao (CR) bound is typically computed to estimate the minimum theoretical variance of a localization estimate. Recently, it has been shown [Makris and Naftali, J. Acoust. Soc. Am. 106, 2 (1999)] that the first-order bias and second-order variance of a general maximum-likelihood estimator can be computed analytically, using higher-order asymptotics in a Taylor series expansion. When applied to the specific case of a matched-field processor with a deterministic signal buried in correlated waveguide noise, it was found that the CR bound underestimates the true theoretical variance for low signal-to-noise ratio (SNR) signals and small sample sizes. Here, the case of a randomized signal in correlated ocean noise is addressed, which represents another facet of the generalized maximum-likelihood estimator problem. Similar to the deterministic case, it is found that the second-order variance term is significant even at $20 \mathrm{~dB}$ SNR, and that the localization bias becomes significant as the SNR approaches zero.

\section{8:30}

3aSP3. Performance analysis for a short vertical array in a shallowwater waveguide. Tainfu Gao, Lan Wang (Inst. of Acoust., Chinese Acad. of Sci., Beijing, PROC), and E. C. Shang (Univ. of Colorado/ NOAA/ETL, Boulder, CO 80303)

Vertical line array (VLA) has been widely used for field data collection in underwater acoustics. The performance of VLA strongly depends on: (1) the signal processing approach used, (2) the environmental knowledge, (3) the signal-to-noise ratio $(\mathrm{S} / \mathrm{N})$, and (4) the configuration of the array (length, position, curvature). In this paper, we concentrate our investigation on the length effect. Theoretical analysis has been done for a short array in a Pekeris shallow-water waveguide. For lower frequency in shallow water, the information provided by a VLA can be described by the capability of mode decomposition, which is determined by the spectrum of the eigenvalue of the "covariance matrix" of the field sampled by the VLA. The approximate decay behavior of the spectrum is calculated analytically for short array. It is shown that the estimated eigenvalues are very close to the exact eigenvalues. The indicator of the performance used in this paper is the number of "S," which represents the dimension of the "signal space," and can be easily determined by the spectrum decay law.

\section{$8: 45$}

3aSP4. Weiss-Weinstein bound for matched-field parameter estimation. Wen Xu and Arthur B. Baggeroer (MIT, Cambridge, MA 02139,wenxu@mit.edu)

Matched-field parameter estimation, including source localization and environmental parameter estimation, is often implemented based on an ambiguity surface. The ambiguity surface is derived from the correlation between the observed signal field and the modeled signal field, and is often characterized by a multimodal structure since the typical signal field is a highly nonlinear function of the embedded parameters. In the presence of noise or other interferences, a high sidelobe level leads to a large error probability in parameter estimation. This sidelobe effect increases as the signal-to-noise ratio (SNR) decreases such that, below a threshold SNR, the estimation mean-square-error (MSE) is dominated by the error around the sidelobes, which is not predicted by the Cramer-Rao bound (CRB). To analyze this effect, the Weiss-Weinstein bound (WWB) is presented for the attainable performance of matched field methods. The bound is expressed in terms of the SNR and an ambiguity function defined based on the Green's function both at the receiver array. An evaluation example 
demonstrates how the ambiguity behavior, particularly the sidelobe structure, affects the bound's evaluation in different SNR regions and leads to the well-known threshold phenomenon in nonlinear parameter estimation.

9:00

3aSP5. Matched-field processing in shallow water in the presence of soliton packets. Catherine Stamoulis (MIT Dept. of Ocean Eng., 77 Massachusetts Ave., Cambridge, MA 02139 and Naval Res. Lab., Washington, DC 20375, caterina@quake.mit.edu), Marshall Orr (Naval Res. Lab., Washington, DC 20375), and Ira Dyer (MIT, Cambridge, MA 02139), and SWARM Group (Naval Res. Lab, Univ. of Delaware, Naval Postgrad. School, Woods Hole Oceanogr. Inst.)

The performance of matched-field processing algorithms in shallow water, in the presence of soliton packets, has been investigated through analysis of data from the SWARM 95 experiment. In particular, acoustic data collected with a vertical array and a moving up-sweep source have been analyzed, in the frequency range $275-350 \mathrm{~Hz}$. The source was placed below the surface mixed layer and its distance from the array varied between 2.5 and $27 \mathrm{~km}$. Source localization using the conventional and minimum variance matched-field algorithms has been performed, in order to assess the robustness of these processors in the presence of environmental mismatch and their degradation as a function of range. The primary result of the analysis is that both processors fail to localize the source accurately, even at the shortest range $(2.5 \mathrm{~km})$. Although broadband processing and inclusion of corrections for the array tilt improved the results, the errors in source range (of the order of $1 \mathrm{~km}$ at short ranges and 5 to $10 \mathrm{~km}$ at long ranges) and depth remain significant. The performance of other matched-field processors is currently investigated.

\section{9:15}

3aSP6. Detection of impulsive signals in shallow water. Peter G. Cable (BBN Technologies, Union Station, New London, CT 06320)

The effect of multipath induced time spread on the detectability of impulsive signals transmitted in shallow water is considered. The effective signal duration and temporal shape of received signal energy, two related features of the pulse spread associated with propagation, were analyzed for the shallow-water coastal environments in the DARPA Area Characterization Tests (ACT I, II, and III). Measured multipath induced time spreads were between 30 and $40 \mathrm{~ms}$ and, for each environment, the integrated received energy was found to be well fitted by an exponential function of the integration time. Using the observed signal energy durations and shapes, a model of energy detection of impulsive signals in shallow water was formulated and will be described. An analysis of expected performance (probability of signal detection for fixed probability of false alarm) as a function of input signal-to-background energy ratio and integration time will be presented.

\section{9:30-9:45 Break}

\section{$9: 45$}

3aSP7. First-order bias and second-order variance of maximum likelihood time delay and Doppler shift estimates. Eran Naftali and Nicholas C. Makris (MIT, 77 Massachusetts Ave., Cambridge, MA 02139)

Exact expressions for the bias and variance of a maximum likelihood time delay and Doppler shift estimate are difficult to obtain analytically. It has become popular in such nonlinear problems to compute limiting bounds on mean-square estimation error, such as the Cramer-Rao bound (CRB), since these are easier to obtain than the true variance. Recently it has been shown [Makris and Naftali, J. Acoust. Soc. Am. 106, 2 (1999)] that the first-order bias and second-order variance of a general maximumlikelihood estimator (MLE) can be computed analytically, using higherorder asymptotics. This approach is applied to the classic radar/sonar problem of estimating the time delay and Doppler shift of a deterministic signal in additive white Gaussian noise. The MLE is shown to be unbiased to first order for this problem. By evaluating the asymptotic expansion for the variance for three specific signal waveforms, Gaussian, LFM, and HFM, it is found that (a) the CRB yields an unrealistically optimistic variance estimate for SNR's lower than 20-30 dB, (b) both first- and second-order time-delay variance terms decrease with increasing signal bandwidth, and (c) both first and second Doppler-shift variance terms of LFM and HFM signals approach constant values as bandwidth increases.

\section{0:00}

3aSP8. Sensitivity analysis of an estimator-correlator. Roger W. Schwenke and Leon H. Sibul (Appl. Res. Lab., The Penn State Univ., P.O. Box 30, State College, PA 16804, rws143@psu.edu)

The estimator-correlator (EC) is a maximum likelihood-sense optimum detector for detection of objects that have multiple random highlights rather than a single deterministic highlight. The EC is also a modelbased signal processor that uses target scattering function as an a priori statistical model. This EC has uses in sonar, medical ultrasound, and multipath communication channels. The receiver operator characteristics (ROCs) of this EC are derived. A sensitivity analysis is derived which computes the change in the ROC curve when there are errors in the $a$ priori estimate of the scattering function. Examples of common scattering function errors and the resulting change in sensitivity are given. [This work has been supported by ONR, Code ONR333, Ms. Khine Latt Program Officer.]

\section{0:15}

3aSP9. Relating modem performance to the ocean channel properties. Michael Porter (Science Applications Intl. Corp., 888 Prospect St., La Jolla, CA 92037, michael.b.porter@ saic.com), Vincent McDonald, Joseph Rice, and Paul Baxley (SPAWAR SSC, San Diego, CA 92152)

The ocean channel plays an important role in the performance of acoustic modems. However, the precise effects of such features as the sea surface, ocean bottom, bubble clouds, and internal waves is not well understood. In many shallow water areas the sound-speed profile varies from upward to downward refracting with the change of seasons enhancing the role of one or the other boundary. Furthermore, incoherent and coherent signaling schemes may be sensitive to different features of the propagation physics. To carefully study these issues, a series of experiments has been planned in diverse sites. The pilot experiment, ModemEx99, was conducted last May near San Diego and has revealed numerous interesting features. In particular, we see that there are strong transitions in the multipath structure with pronounced focal regions and shadow zones. These features correlate closely with areas of good and poor modem performance, respectively. This talk will interpret these results in light of the measured channel impulse response and predictions from broadband channel simulators.

\section{0:30}

3aSP10. Coherent underwater digital communication during LWAD 99-1 experiment: Performance and analysis. Azmi Al-Kurd (Naval Res. Lab., 4555 Overlook Ave. SW, Washington, DC 20375-5320, a.alkurd@ieee.org)

Underwater acoustic communications data using phase-modulated signals were collected during the Littoral Warfare Advanced Development (LWAD 99-1) experiment. The signals were projected from a drifting and a towed source. Data of several band rates were transmitted and used to study the temporal and spatial variation of the acoustic impulse response of the ocean and to evaluate the performance of a phase coherent digital communication algorithm. Postexperimental analysis showed that the experiment site is a harsh environment for coherent digital communication. The oceanic channel impulse response is very complex and represents a dynamic and extended multipath structure (changes in seconds). Also, the first arrival is weaker than the later arrivals (most of the time). In addition to the environmental limitations the receiver has to overcome system limitations and operate in a low signal-to-noise ratio conditions. Spatial and temporal diversity are implemented to improve the algorithm performance. The objective is to determine the characteristics of signal propa- 
gation in littoral environment, and to determine whether and how these characteristics affect the bit-error-rate. The results from this experiment and future experiments will serve as guidance in the design of a reliable coherent digital communication system.

\section{0:45}

3aSP11. Quantifying data information content in matched-field inversion. Stan E. Dosso, Edward Chapin, and Michael J. Wimut (School of Earth and Ocean Sci., Univ. of Victoria, Victoria, BC V8W 3P6, Canada, sdosso@uvic.ca)

In matched-field inversion, geoacoustic properties and/or source position are determined by minimizing an objective function that represents the mismatch between measured and modeled acoustic fields. As this is a strongly nonlinear problem, linearized inversion and appraisal algorithms do not apply. A problem of practical importance is to quantify how the inversion results are influenced by data factors such as (i) signal-to-noise level, (ii) number of frequencies included in the objective function, (iii) whether multiple frequencies are summed coherently or incoherently, and (iv) sensor array configuration. This problem is examined here by applying a fully nonlinear analysis based on Gibbs sampling to construct posteriori probability distributions (PPDs) for the unknown model parameters. Constructing PPDs for different realizations of the above factors allows their effect on the solution of the matched-field inverse problem to be quantified and exploited.
11:00

3aSP12. Extraction of modal amplitudes for the analysis of two- and three-dimensional underwater sound propagation. Joseph M. Riley and Stewart A. L. Glegg (Florida Atlantic Univ., Ctr. for Acoust. and Vib., Dept. of Ocean Eng., Bldg. \#36, Rm. 190, 777 W. Glades Rd., Boca Raton, FL 33431)

Experimental measurements have been conducted over a laboratory scale model of the Santa Lucia Escarpment for the purpose of analyzing two- and three-dimensional underwater sound propagation. Transmission loss (TL) was measured as a function of depth at a series of ranges in the downslope direction of the model (referred to as depth profile measurements) and TL was also measured over a finite area in the horizontal plane for fixed source and receiver depths (referred to as two-dimensional surveys). The two-dimensional surveys show across slope interference, which may be indicative of bathymetric refraction, and the modal structure of the depth profile measurements appears to change as a function of downslope range (a result which may be caused by mode coupling). In order to investigate the interference observed in the two-dimensional surveys and also determine if the range-dependent variation in the depth profile measurements is being caused by mode coupling, an inversion algorithm was applied to measurements of the complex pressure field for the purpose of extracting the modal amplitudes. Analysis of the experimentally extracted modal amplitudes indicates that mode coupling and bathymetric refraction contributed significantly to the acoustic pressure field. [Work supported by ONR.]

\title{
Session 3pAA
}

\section{Architectural Acoustics: Classroom Acoustics (Poster Session)}

\author{
Brandon D. Tinianow, Chair \\ Acoustical Laboratory, Johns Manville, 10100 West Ute Avenue, Littleton, Colorado 80162
}

\section{Contributed Papers}

\begin{abstract}
All posters will be on display from 1:00 p.m. to 3:00 p.m. All contributors will be at their posters from 1:00 p.m. to 3:00 p.m.
\end{abstract}

\begin{abstract}
3pAA1. Eliminating acoustical barriers to learning in classroomsCase study of window ventilator noise. Bennett M. Brooks (Brooks Acoust. Corp., 27 Hartford Turnpike, Vernon, CT 06066, bbrooks@brooks-acoustics.com)

Room ventilation systems have long been a major cause of noise in classrooms. The recent drive for energy efficiency has motivated schools to partner with utility companies to replace aging central HVAC systems with individual room heat pump window ventilator units for space heating and cooling. An unfortunate consequence is that these window ventilators are significant noise sources. A typical window unit can produce 70 $\mathrm{dB}(\mathrm{A})$, or more, at $1 \mathrm{~m}$. Clearly, this is unacceptable. Either ventilator manufacturers must commit to reduce unit noise output by at least $30 \mathrm{~dB}$, or school designers must abandon the wall ventilator option in favor of quiet central HVAC installations.
\end{abstract}

3pAA2. Improving existing classroom sound isolation for advance media capabilities. Dana Hougland (A CODA Acoustic, LLC 9603 E. Orchard Dr., Englewood, CO 80111, acoda@aol.com)

A series of tests was conducted to assess the incremental improvement of the noise reduction and sound transmission loss between adjacent classrooms with various impovements to the demising construction. Classrooms were originally constructed without full height walls. Tests were conducted before modifications were implemented and after each modification was completed. The investigation was conducted as part of a larger classroom improvement project design to bring advanced media capabilities into 60 college classrooms campus wide. The results of the testing program are presented. 